\title{
Multiequilibrium Game of Timing and Competition of Gas Pipeline Projects
}

G.Klaassen, A.V. Kryazhimskii, and A.M. Tarasyev

RR-04-002

February 2004

Reprinted from Journal of Optimization and Applications, 120(1):147-179 (2004). 
Research Reports, which record research conducted at IISA, are independently reviewed before publication. Views or opinions expressed herein do not necessarily represent those of the Institute, its National Member Organizations, or other organizations supporting the work.

Reprinted with permission from Journal of Optimization and Applications, 120(1):147-179 (2004).

Copyright (C) 2004 Plenum Publishing Corporation.

All rights reserved. No part of this publication may be reproduced or transmitted in any form or by any means, electronic or mechanical, including photocopy, recording, or any information storage or retrieval system, without permission in writing from the copyright holder. 


\title{
Multiequilibrium Game of Timing and Competition of Gas Pipeline Projects ${ }^{1}$
}

\author{
G. KlaAssen, ${ }^{2}$ A. V. Kryazhimski, ${ }^{3}$ and A. M. Tarasyev ${ }^{4}$ \\ Communicated by F. L. Chernousko
}

\begin{abstract}
The paper addresses the issue of the optimal investments in innovations with strong long-tern aftereffects. As an example, investments in the construction of gas pipelines are considered. The most sensitive part of an investment project is the choice of the commercialization time (stopping time), i.e., the time of finalizing the construction of the pipeline. If several projects compete on the market, the choices of the commercialization times determine the future structure of the market and thus become especially important. Rational decisions in this respect can be associated with Nash equilibria in a game between the projects. In this game, the total benefits gained during the pipelines life periods act as payoffs and the commercialization times as strategies. The goal of this paper is to characterize multiequilibria in the game of timing. The case of two players is studied in detail. A key point in the analysis is the observation that, for all players, the best response commercialization times concentrate at two instants that are fixed in advance. This reduces decisionmaking to choosing between two fixed investment policies (fast and slow) with the prescribed commercialization times. A description of a simple algorithm that finds all the Nash equilibria composed of fast and slow scenarios concludes the paper.
\end{abstract}

Key Words. Optimal stopping problem, game of timing, multiequilibria, best reply curves, econometric estimation.

\footnotetext{
'The second author was partially supported by the Russian Fund for Fundamental Research, Grant 03-01-00737. The third author was partially supported by the Russian Fund for Fundamental Research, Grant 02-01-00769, and by the Program for the Sponsorship of Leading Scientific Schools, Grant 00-15-96057.

${ }^{2}$ Reserach Scholar, International Institute for Applied Systems Analysis, Laxenburg, Austria.

${ }^{3}$ Professor, Steklov Mathematical Institute, Moscow, Russia; Coordinator of the Dynamic Systems Project, International Institute for Applied Systems Analysis, Laxenburg, Austria.

${ }^{4}$ Leading Researcher, Institute of Mathematics and Mechanics, Ekaterinburg, Russia; Research Scholar, International Institute for Applied Systems Analysis, Laxenburg, Austria.
} 


\section{Introduction}

When several large-scale gas pipeline projects compete for a new gas market, the choices of the commercialization times (stopping times), i.e., the times of finalizing the construction of the pipelines, determine the future structure of the market and thus become especially important. In Ref. 1, which motivated the present study, a detailed pipeline model based on classical patterns of mathematical economics (see Refs. 2--3) was designed and a best reply dynamic adaptation algorithm originating from the theory of evolutionary games (see Refs. 4-9) was used to estimate numerically the commercialization times for the pipeline projects competing nowadays for the Turkey gas market.

Rational choices of the commercialization times can be viewed as Nash equilibria in a game between the projects. In the present paper, we study the structure of this game. In order to make the model easily tractable in terms of game theory (see Refs. 10-11), we introduce several simplifying assumptions; in particular, we reduce the number of competing projects to two. A background in the analysis of gas infrastructures (see Ref. 12) and problems of optimal timing (see Refs. 13-14) is employed.

The model takes into account the stages of construction and exploitation of the gas pipelines. In each level, the model is optimized and estimated using appropriate techniques of the theory of optimal control and theory of differential games (see Refs. 15-17). At the stage of exploitation, as gas supply policies compete on the market, decisionmaking is relatively clear: the competitors search for an equilibrium supply at any instant. Therefore, we focus on the stage of construction, at which investment policies compete and decisionmaking is concerned with strong long-term aftereffects. The competitors interact through choosing their commercialization times. A proper individual choice is the best response to the choices of the other competitor. Therefore, a pair of commercialization times is suitable to every competitor if and only if the commercialization time of every competitor responds best to the commercialization time of the other competitor. Such situations constitute Nash equilibria in the game under consideration. In this game, the total benefits gained during the pipelines life periods act as payoffs and the commercialization times act as strategies. Our goal is to characterize the equilibria in this game, which will be referred to further as game of timing.

In Section 2, we describe the general two-player game of timing, in which the cost and benefit functions determining the players payoffs are not specified. In Section 3, we find the Nash equilibria in the game. A key point in the analysis is the observation that, for all players, the best response commercialization times concentrate at two instants that are fixed in advance. 
This reduces decisionmaking to choosing between two fixed investment policies (fast and slow) with the prescribed commercialization times.

In Section 4, we describe an algorithm that finds all the Nash equilibria in the game of timing. In Section 5, we study the game of timing for the model of operation of gas pipelines which was described in Ref. 1.

In Section 6, we give results of the model-based analysis for two case studies: competing gas pipeline projects in the Caspian region and planned pipeline routes to the gas market in China. Finally, Section 7 contains the proofs of the propositions formulated in Section 4.

\section{Game of Timing}

In this section, we construct a game-theoretic model of competition of two gas pipeline projects. We call it the game of timing. The pipelines are expected to operate at the same market. We associate players 1 and 2 with the investors/managers of projects 1 and 2, respectively. Assuming that the starting time for making investments is $t=0$, we consider virtual positive commercialization times $t_{1}$ and $t_{2}$ of projects 1 and 2 (i.e., the final times of the construction of the pipelines). Given a (virtual) commercialization time $t_{i}$ player $i, i=1,2$, can estimate the cost $C_{i}\left(t_{i}\right)$ for finalizing project $i$ at time $t_{i}$. The positive-valued cost functions $C_{i}\left(t_{i}\right), i=1,2$, are defined on the positive half axis. The following assumption will simplify our analysis.

Assumption 2.1. For each player $i$, the cost function $C_{i}\left(t_{i}\right)$ is smooth (continuously differentiable), monotonically decreasing, and convex.

A formal interpretation of Assumption 2.1 is that the derivative $C_{i}^{\prime}\left(t_{i}\right)=d C_{i}\left(t_{i}\right) / d t_{i}$ is negative and increasing. A substantial interpretation is that the cost of the project falls down as the project commercialization period is prolonged; moreover, the longer is the commercialization period, the less sensitive, with respect to its prolongations, is the rate of cost reduction. In what follows, the rate of cost reduction for player $i$ is understood as the positive-valued monotonically decreasing function

$$
a_{i}\left(t_{i}\right)=-C_{i}^{\prime}\left(t_{i}\right) \text {. }
$$

Let us argue for player 1 as the manager of pipeline 1 . At any time $t>0$, the price of gas and costs for extraction and transportation of gas determine the benefit rate $b_{1}(t)$ of player 1 (note that this benefit rate is virtual, because $t$ may precede the actual commercialization time of project 1$)$. The costs for extraction and transportation of gas do not depend on the state of project 2 , whereas the price of gas depends on the presence (absence) of player 2 on the 
marketplace. In the situation where both players operate on the market, the price of gas should obviously be smaller compared to the situation where player 1 occupies the market solely. Hence, the benefit rate $b_{1}(t)$ may take two values, $b_{11}(t)$ and $b_{12}(t)$, with

$$
b_{11}(t)>b_{12}(t) \text {. }
$$

We call $b_{11}(t)$ the upper benefit rate and $b_{12}(t)$ the lower benefit rate of player 1 at time $t$. At time $t$ (which virtually follows the commercialization time of player 1 ), player 1 virtually gets $b_{11}(t)$, if player 2 does not operate on the market, and gets $b_{12}(t)$, if player 2 operates on the market. Similarly, we introduce the upper and lower benefit rates of player 2 at time $t, b_{21}(t)$ and $b_{22}(t)$, with

$$
b_{21}(t)>b_{22}(t)
$$

A time $t$, player 2 gets $b_{21}(t)$, if player 1 does not operate on the market, and gets $b_{22}(t)$, otherwise. We assume that the positive-valued upper and lower benefit rates $b_{i 1}(t)$ and $b_{i 2}(t), i=1,2$, are continuous functions defined on the positive half axis. We introduce also the following assumption.

Assumption 2.2. For every player $i, i=1,2$, the graph of the rate of cost reduction $a_{i}(t)$ intersects the graph of the upper benefit rate $b_{i 1}(t)$ from above at a unique point $t_{i}>0$ and stays below it afterward; similarly, the graph of $a_{i}(t)$ intersects the graph of $b_{i 2}(t)$ from above at a unique point $t_{i}^{+}>0$ and stays below it afterward; more accurately,

$$
\begin{array}{lll}
a_{i}(t)>b_{i 1}(t) \text { for } 0<t<t_{i}^{-}, & a_{i}\left(t_{i}^{-}\right)=b_{i 1}\left(t_{i}^{-}\right), & a_{i}(t)<b_{i 1}(t) \text { for } t>t_{i}^{-}, \\
a_{i}(t)>b_{i 2}(t) \text { for } 0<t<t_{i}^{+}, & a_{i}\left(t_{i}^{+}\right)=b_{i 2}\left(t_{i}^{+}\right), & a_{i}(t)<b_{i 2}(t) \text { for } t>t_{i}^{+} .
\end{array}
$$

Remark 2.1. Assumption 2.2 implies in particular that, if $t>0$ is sufficiently small, the rate of cost reduction $a_{i}(t)$ is greater than the upper benefit rate $b_{i 1}(t)$; if $t>0$ is sufficiently large, the rate of cost reduction $a_{i}(t)$ is smaller than the lower benefit rate $b_{i 2}(t)$.

Remark 2.2. Since $a_{i}(t)$ is decreasing and $b_{i 1}(t)>b_{i 2}(t)$ [see (2) and (3)], we have

$$
t_{i}^{-}<t_{i}^{+}
$$

The graph of the rate of cost reduction $a_{i}(t)$ and the graphs of the upper and lower benefit rates $b_{i 1}(t)$ and $b_{i 2}(t)$ are shown schematically in Fig. 1.

The fact that $t_{2}$ is the commercialization time of player 2 implies that player 2 does not operate on the market at any time $t<t_{2}$ and operates on 


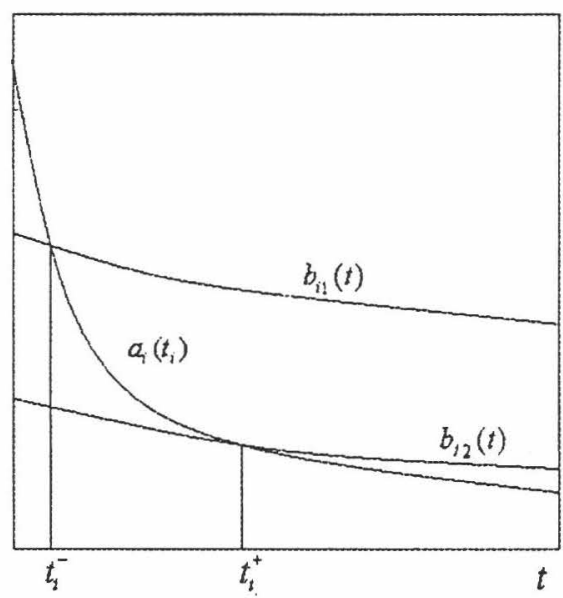

Fig. 1. Rate of cost reduction $a_{i}(t)$ and upper and lower benefit rates $b_{i 1}(t)$ and $b_{i 2}(t)$.

the market at every time $t \geq t_{2}$. Accordingly, the benefit rate $b_{1}(t)$ of player 1 equals $b_{11}(t)$ for $t<t_{2}$ and equals $b_{12}(t)$ for $t \geq t_{2}$. We stress the dependence of $b_{1}(t)$ on $t_{2}$ and write $b_{1}\left(t \mid t_{2}\right)$ instead of $b_{1}(t)$. Thus, given a commercialization time $t_{2}$ of project 2 , the benefit rate of player 1 is found as

$$
b_{1}\left(t \mid t_{2}\right)= \begin{cases}b_{11}(t), & \text { if } t<t_{2}, \\ b_{12}(t), & \text { if } t \geq t_{2} .\end{cases}
$$

Similarly, a commercialization time $t_{1}$ of project 1 determines the benefit rate of player 2 as

$$
b_{2}\left(t \mid t_{1}\right)= \begin{cases}b_{21}(t), & \text { if } t<t_{1}, \\ b_{22}(t), & \text { if } t \geq t_{1} .\end{cases}
$$

The graphs of the benefit rates $b_{1}\left(f \mid t_{2}\right)$ and $b_{2}\left(t \mid t_{1}\right)$ are shown schematically in Fig. 2.

Given a commercialization time $t_{1}$ of player 1 , and a commercialization time $t_{2}$ of player 2 , the total benefits of players 1 and 2 are represented by the integrals

$$
\begin{aligned}
& B_{1}\left(t_{1}, t_{2}\right)=\int_{t_{1}}^{\infty} b_{1}\left(t \mid t_{2}\right) d t, \\
& B_{2}\left(t_{1}, t_{2}\right)=\int_{t_{2}}^{\infty} b_{2}\left(t \mid t_{1}\right) d t,
\end{aligned}
$$

respectively. 


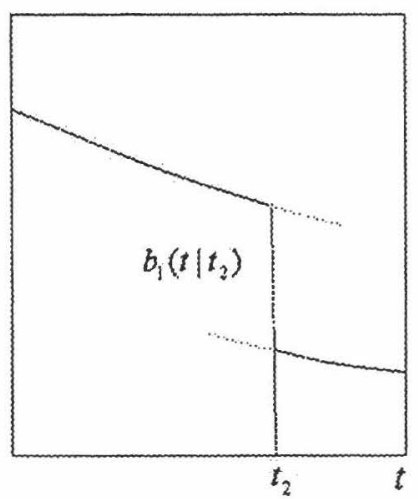

(a)

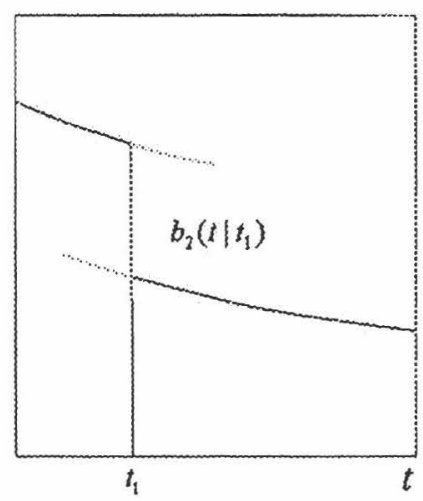

(b)

Fig. 2. (a) Benefit rate of player 1. (b) Benefit rate of player 2 .

We make the following natural assumption.

Assumption 2.3. For every positive time $t_{1}$ and every positive time $t_{2}$, the integrals $B_{1}\left(t_{1}, t_{2}\right)$ and $B_{2}\left(t_{1}, t_{2}\right)$ are finite.

Remark 2.3. Assumption 2.3 is equivalent to the following: for every positive time $t_{1}$ and every positive time $t_{2}$, the integrals $\int_{t_{2}}^{\infty} b_{12}(t) d t$, $\int_{i_{1}}^{\infty} b_{22}(t) d t$ are finite.

Given a commercialization time $t_{1}$ of player 1 and a commercialization time $t_{2}$ of player 2 , the total profit of player $i$ is defined as

$$
P_{i}\left(t_{1}, t_{2}\right)=-C_{i}\left(t_{i}\right)+B_{i}\left(t_{1}, t_{2}\right) .
$$

We are ready to define the game of timing for players 1 and 2 in line with the standards of game theory (see Ref. 11). In the game of timing, the strategies of player $i, i=1,2$, are the positive (virtual) commercialization times $t_{i}$, for project $i$; the payoff to player $i$, thanks to the strategies $t_{1}$ and $t_{2}$ of players 1 and 2 , respectively, is the total profit $P_{i}\left(t_{1}, t_{2}\right)$.

\section{Nash Equilibria}

According to the standard terminology of game theory, a strategy $t_{1}^{*}$ of player 1 is said to be a best response of player 1 to a strategy $t_{2}$ of player 2 if $t_{1}^{*}$ maximizes the payoff $P_{1}\left(t_{1}, t_{2}\right)$ to player 1 over the set of all strategies $t_{1}$ 
of player 1 ,

$$
P_{1}\left(t_{1}^{*}, t_{2}\right)=\max _{t_{1}>0} P_{1}\left(t_{1}, t_{2}\right) .
$$

Similarly, a strategy $t_{2}^{*}$ of player 2 is said to be a best response of player 2 to a strategy $t_{1}$ of player 1 if $t_{2}^{*}$ maximizes the payoff $P_{2}\left(t_{1}, t_{2}\right)$ to player 2 over the set of all strategies $t_{2}$ of player 2,

$$
P_{2}\left(t_{1}, t_{2}^{*}\right)=\max _{t_{2}>0} P_{2}\left(t_{1}, t_{2}\right) \text {. }
$$

Any pair $\left(t_{1}^{*}, t_{2}^{*}\right)$, where $t_{1}^{*}$ is a strategy of player 1 and $t_{2}^{*}$ a strategy of player 2, is said to be a Nash equilibrium in the game of timing if $t_{1}^{*}$ is a best response of player 1 to $t_{2}^{*}$ and $t_{2}^{*}$ is a best response of player 2 to $t_{1}^{*}$. Our goal is to characterize the Nash equilibria in the game of timing.

We start with a simple observation concerned with the dependence of the player payoff on the strategy of the other player. For example, let us consider the payoff $P_{1}\left(t_{1}, t_{2}\right)$ to player 1 . The differentiation of $P_{1}\left(t_{1}, t_{2}\right)$ with respect to $t_{1}$ yields

$$
\begin{aligned}
\partial P_{1}\left(t_{1}, t_{2}\right) / \partial t_{1} & =a_{1}\left(t_{1}\right)-b_{1}\left(t_{1} \mid t_{2}\right) \\
& = \begin{cases}a_{1}\left(t_{1}\right)-b_{11}\left(t_{1}\right), & \text { if } t_{1}<t_{2}, \\
a_{1}\left(t_{1}\right)-b_{12}\left(t_{1}\right), & \text { if } t_{1}>t_{2} .\end{cases}
\end{aligned}
$$

Here, we have used (10), (1), (8), and (7). Note that the above partial derivative exists and is continuous at any $t_{1}>0$ except for $t_{1}=t_{2}$. Geometrically, (11) means that $P_{1}\left(t_{1}, t_{2}\right)$ grows in $t_{1}$ on the time intervals where the graph of $a_{1}\left(t_{1}\right)$ lies above the graph of $b_{1}\left(t_{1} \mid t_{2}\right)$ an declines in $t_{1}$ on the time intervals where the graph of $a_{1}\left(t_{1}\right)$ lies below the graph of $b_{1}\left(t_{1} \mid t_{2}\right)$.

Let us take two arbitrary strategies of player $2, t_{21}$ and $t_{22}>t_{21}$. As (11) shows,

$$
\partial P_{1}\left(t_{1}, t_{22}\right) / \partial t_{1}=\partial P_{1}\left(t_{1}, t_{21}\right) / \partial t_{1}
$$

for $t_{1}<t_{21}$ and $t_{1}>t_{22}$, and

$$
\partial P_{1}\left(t_{1}, t_{22}\right) / \partial t_{1}=\partial P_{1}\left(t_{1}, t_{21}\right) / \partial t_{1} \cdots\left[b_{11}\left(t_{1}\right) \cdots b_{12}\left(t_{1}\right)\right],
$$

for $t_{21}<t_{1}<t_{22}$. Recall that

$$
b_{11}\left(t_{1}\right) \cdots b_{12}\left(t_{1}\right)>0 \text {; }
$$

see (2). We have stated that, beyond the time interval located between $t_{21}$ and $t_{22}, P_{1}\left(t_{1}, t_{22}\right)$ and $P_{1}\left(t_{1}, t_{21}\right)$ have the same rate in $t_{1}$ and, that within this time interval, $P_{1}\left(t_{1}, t_{22}\right)$ declines in $t_{1}$ faster than $P_{1}\left(t_{1}, t_{21}\right)$. Thanks to $(8)$ and ( 7$)$,

$$
P_{1}\left(t_{1}, t_{22}\right)=P_{1}\left(t_{1}, t_{21}\right), \quad \text { for } t_{1} \geq t_{22} \text {. }
$$




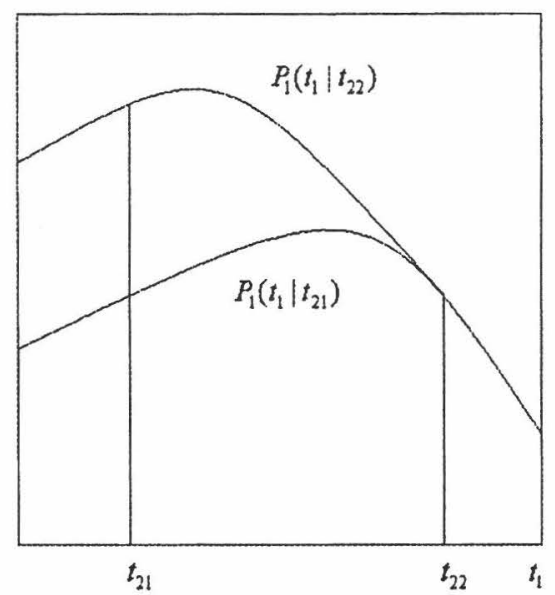

Fig. 3. Payoff $P_{1}\left(t_{1}, t_{2}\right)$ for $t_{2}=t_{21}$ and $t_{2}=t_{22}>t_{21}$.

Therefore,

$$
P_{1}\left(t_{1}, t_{22}\right)>P_{1}\left(t_{1}, t_{21}\right), \quad \text { for } t_{1}<t_{22} \text {. }
$$

Let us sum up the previous arguments in the following statement.

Proposition 3.1. For every $t_{1}>0$, the payoff $P_{1}\left(t_{1}, t_{2}\right)$ to player 1 increases in $t_{2}$; moreover, given a $t_{21}>0$ and a $t_{22}>t_{21}$, one has $P_{1}\left(t_{1}, t_{22}\right)=$ $P_{1}\left(t_{1}, t_{21}\right)$ for $t_{1} \geq t_{22}$ and $P_{1}\left(t_{1}, t_{22}\right)>P_{1}\left(t_{1}, t_{21}\right)$ for $t_{1}<t_{22}$.

The graphs of $P_{1}\left(t_{1}, t_{2}\right)$ for $t_{2}=t_{21}$ and $t_{2}=t_{22}>t_{21}$ are shown in Fig. 3 .

A symmetric argument leads to a similar observation for player 2 .

Proposition 3.2. For every $t_{2}>0$, the payoff $P_{2}\left(t_{1}, t_{2}\right)$ to player 2 increases in $t_{1}$; moreover, given a $t_{11}>0$ and a $t_{12}>t_{11}$, one has $P_{2}\left(t_{12}, t_{2}\right)=$ $P_{2}\left(t_{11}, t_{2}\right)$ for $t_{2} \geqslant t_{12}$ and $P_{2}\left(t_{12}, t_{2}\right)>P_{2}\left(t_{11}, t_{2}\right)$ for $t_{2}<t_{12}$.

Remark 3.1. The fact stated in Propositions 3.1 and 3.2 is intuitively clear: for the investor/manager of a gas pipeline project, any prolongation of the commercialization period of the competing project is profitable.

Now, let us find the best responses (the best reply curve) of player 1 to a given strategy $t_{2}$ of player 2 .

It is easy enough to identify the intervals of growth and decline of the payoff $P_{1}\left(t_{1}, t_{2}\right)$ as a function of $t_{1}$. We use $(11)$ and refer to the points $t_{1}^{-}$and $t_{1}^{+}$, at which the graph of $a_{1}(t)$ intersects the graphs of $b_{11}(t)$ and $b_{12}(t)$; see (4), (5), and Fig. 2. 


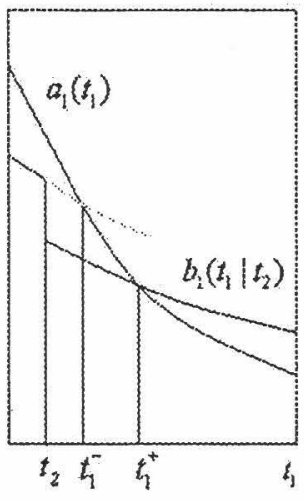

(8)

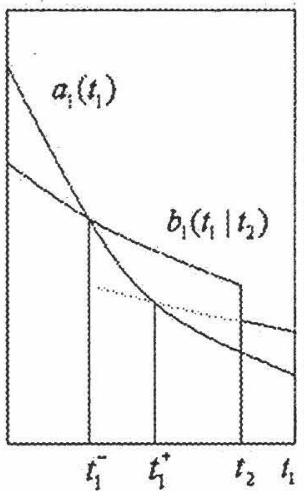

(b)

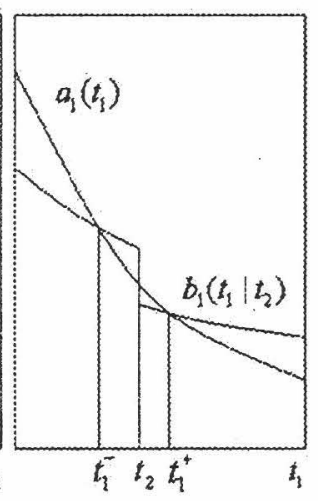

(c)

Fig. 4. Functions $a_{1}\left(t_{1}\right)$ and $b_{1}\left(t_{1} \mid t_{2}\right)$ for (a) $t_{2} \leq t_{1}^{-}$, (b) $t_{2} \geq t_{1}^{+}$, (c) $t_{1} \leq t_{2} \leq t_{1}^{+}$.

Assume first that $t_{2} \leq t_{1}^{-}$; recall that $t_{1}<t_{1}^{*}[\operatorname{see}(6)]$. Then, as (4), (5), and Fig. 2 show, the graph of $a_{1}\left(t_{1}\right)$ lies above the graph of $b_{1}\left(t_{1} \mid t_{2}\right)$ for $t_{1}<t_{1}^{+}$and lies below it for $t_{1}>t_{1}^{+}$; at $t_{1}=t_{1}^{+}$, the graphs intersect. Figure 4(a) illustrates the relations between the graphs.

Due to (11), $\partial P_{1}\left(t_{1}, t_{2}\right) / \partial t_{1}$ is positive for $t_{1}<t_{1}^{+}, t_{1} \neq t_{2}$, and negative for $t_{1}>t_{1}^{+}$. Therefore, $t_{1}=t_{1}^{+}$is the unique maximizer of $P_{1}\left(t_{1}, t_{2}\right)$ in the set of all positive $t_{1}$; in other words, $t_{1}^{+}$is the single best response of player 1 to strategy $t_{2}$ of player 2 .

Let us assume that $t_{2} \geq t_{1}^{+}$. Then, (4), (5), and Fig. 2 show that the graph of $a_{1}\left(t_{1}\right)$ lies above the graph of $b_{1}\left(t_{1} \mid t_{2}\right)$ for $t_{1}<t_{1}$, and lies below it for $t_{1}>t_{1}$; at $t_{1}=t_{1}^{+}$, the graphs intersect. Figure 4 (b) illustrates the relations between the graphs. Due to $(11), \partial P_{1}\left(t_{1}, t_{2}\right) / \partial t_{1}$ is positive for $t_{1}<t_{1}^{-1}$ and negative for $t_{1}>t_{1}^{-}, t_{1} \neq t_{2}$. Hence, $t_{1}=t_{1}^{-}$is the unique maximizer of $P_{1}\left(t_{1}, t_{2}\right)$ in the set of all positive $t_{1}$; i.e., $t_{1}$ is the single best response of player 1 to $t_{2}$.

Now, let $t_{2}$ lie in the interval $\left[t_{1}, t_{1}^{+}\right]$. Then, (4), (5), and Fig. 2 show that the graph of $a_{1}\left(t_{1}\right)$ lies above the graph of $b_{1}\left(t_{1} \mid t_{2}\right)$ for $t_{1}<t_{1}$, lies below it for $t_{1}<\mathrm{t}_{1}<t_{2}$, lies again above the graph of $b_{1}\left(t_{1} \mid t_{2}\right)$ for $t_{2}<t_{1}<t_{1}^{+}$, and lies again below it for $t_{1}>t_{1}^{+}$. Figure 4(c) illustrates the relations between the graphs. Thanks to (11), we conclude that $P_{1}\left(t_{1}, t_{2}\right)$, as a function of $t_{1}$, strictly decreases on the interval $\left(0, t_{1}\right)$, strictly decreases on the interval $\left(t_{1}, t_{2}\right)$, strictly increases on the interval $\left(t_{2}, t_{1}^{+}\right)$, and strictly decreases on the interval $\left(t_{1}^{+}, \infty\right)$. Therefore, the maximizers of $P_{1}\left(t_{1}, t_{2}\right)$ in the set of all positive $t_{1}$, i.e., the best responses of player 1 to $t_{2}$, are restricted to the two-element set $\left\{t_{1}, t_{1}^{+}\right\}$.

Let us identify the actual maximizers in this set. We refer to Proposition 3.1. Suppose that $t_{2}<t_{1}^{+}$. Set $t_{1}=t_{1}^{+}, t_{21}=t_{2}$, and $t_{22}=t_{1}^{+}$. We see that 
$t_{1}=t_{22}>t_{21}$. By Proposition 3.1,

$$
P_{1}\left(t_{1}, t_{22}\right)=P_{1}\left(t_{1}, t_{21}\right)
$$

or

$$
P_{1}\left(t_{1}^{+}, t_{1}^{+}\right)=P_{1}\left(t_{1}^{+}, t_{2}\right)
$$

Since $P_{1}\left(t_{1}^{+}, t_{2}\right)$ is continuous in $t_{2},(12)$ holds for $t_{2}=t_{1}^{+}$as well. Now, we take arbitrary $t_{21}$ and $t_{22}>t_{21}$ in the interval $\left[t_{1}^{+}, t_{1}^{+}\right]$. By Proposition 3.1,

$$
P_{1}\left(t_{1}, t_{22}\right)>P_{1}\left(t_{1}, t_{21}\right)
$$

Therefore, $P_{1}\left(t_{1}, t_{2}\right)$ strictly increases in $t_{2}$ on $\left[t_{1}^{+}, t_{2}^{+}\right]$. Consider the function

$$
p\left(t_{2}\right)=P_{1}\left(t_{1}^{-}, t_{2}\right)-P_{1}\left(t_{1}^{+}, t_{2}\right),
$$

defined on $\left[t_{1}^{-}, t_{1}^{+}\right]$. By (12), we have

$$
p\left(t_{2}\right)=P_{1}\left(t_{1}^{-}, t_{2}\right)-P_{1}\left(t_{1}^{+}, t_{1}^{+}\right)
$$

for all $t_{2}$ in the interval $\left[t_{1}^{+}, t_{2}^{\dagger}\right]$. As long as $P_{1}\left(t_{1}, t_{2}\right)$ strictly increases in $t_{2}$ on $\left[t_{1}^{-}, t_{1}^{+}\right], p\left(t_{2}\right)$ strictly increases on $\left[t_{1}^{+}, t_{2}^{+}\right]$. Earlier, we have stated that $t_{1}^{+}$is the single best response of player 1 to any $t_{2} \leq t_{1}$; in particular, this holds for $t_{2}=t_{1}$, i.e.,

$$
P_{1}\left(t_{1}^{+}, t_{1}^{-}\right)>P_{1}\left(t_{1}^{-}, t_{1}^{-}\right)
$$

Hence,

$$
p\left(t_{1}^{-}\right)=P_{1}\left(t_{1}^{-}, t_{1}^{-}\right)-P_{1}\left(t_{1}^{+}, t_{1}^{-}\right)<0 .
$$

Earlier, we have stated that $t_{1}^{-}$is the single best response of player 1 to any $t_{2} \geq t_{1}^{+}$; in particular, this holds for $t_{2}=t_{1}^{+}$, i.e.,

$$
P_{1}\left(t_{1}^{-}, t_{1}^{+}\right)>P_{1}\left(t_{1}^{+}, t_{1}^{+}\right) \text {. }
$$

Hence,

$$
p\left(t_{1}^{+}\right)=P_{1}\left(t_{1}, t_{1}^{+}\right)-P_{1}\left(t_{1}^{+}, t_{1}^{+}\right)>0
$$

We have found that $p\left(t_{2}\right)$ takes a negative value at the left endpoint of the interval $\left[t_{1}, t_{1}^{+}\right]$and a positive value at the right endpoint of this interval. Since $p\left(t_{2}\right)$ is continuous, there exists a $\hat{t}_{2}$ in the interior of $\left[t_{1}^{-}, t_{2}^{+}\right]$, for which $p\left(\hat{t}_{2}\right)=0$. The fact that $p\left(t_{2}\right)$ strictly increases on $\left[t_{1}^{-}, t_{1}^{+}\right]$implies that the point $\hat{t}_{2}$ is unique; i.e., $p\left(t_{2}\right)<0$ for $t_{1} \leq t_{2}<\hat{t}_{2}$ and $p\left(t_{2}\right)>0$ for $t_{1} \geq t_{2}>\hat{t}_{2}$. By the definition of $p\left(t_{12}\right)$ and (13), we have

$$
\begin{array}{ll}
P_{1}\left(t_{1}^{-}, \hat{t}_{2}\right)=P_{1}\left(t_{1}^{+}, \hat{t}_{2}\right), & \\
P_{1}\left(t_{1}^{-}, t_{2}\right)<P_{1}\left(t_{1}^{+}, t_{2}\right), & \text { for } t_{1}^{-} \leq t_{2}<\hat{t}_{2}, \\
P_{1}\left(t_{1}, t_{2}\right)<P_{1}\left(t_{1}^{+}, t_{2}\right), & \text { for } t_{1}^{+} \geq t_{2}>\hat{t}_{2} .
\end{array}
$$


Earlier, we have stated that all the best responses of player 1 to $t_{2}$ lie in the two-element set $\left\{t_{1}^{-}, t_{1}^{+}\right\}$. Therefore, we conclude that, if $t_{2}=\hat{t}_{2}$, player 1 has two best responses, $t_{1}^{-}$and $t_{1}^{+}$, to $t_{2}$; if $t_{1}^{-} \leq t_{2}<\hat{t}_{2}$, the unique best response of player 1 to $t_{2}$ is $t_{1}^{+}$; and if $t_{1}^{+} \geq t_{2}>\hat{t}_{2}$, the unique best response of player 1 to $t_{2}$ is $t_{1}$. Recall that the best response of player 1 to $t_{2}$ is $t_{1}^{+}$if $t_{2}<t_{1}$, and $t_{1}$ if $t_{2}>t_{1}^{+}$.

We summarize the above considerations as follows.

Proposition 3.3. In the interval $\left(t_{1}^{-}, t_{1}^{+}\right)$, there exists a unique point $\hat{t}_{2}$ such that

$$
P_{1}\left(t_{1}, \hat{t}_{2}\right)=P_{1}\left(t_{1}^{+}, \hat{t}_{2}\right)
$$

The set of all best responses of player 1 to $\hat{t}_{2}$ is $\left\{t_{1}, t_{1}^{+}\right\}$. If $0<t_{2}<\hat{t}_{2}$, then the unique best response of player 1 to $t_{2}$ is $t_{1}^{+}$. If $t_{2}>\hat{t}_{2}$, then the unique best response of player 1 to $t_{2}$ is $t_{1}^{-}$.

We call $t_{1}^{-}$the fast choice of player 1 and $t_{1}^{+}$the slow choice of player 1 . Proposition 3.3 claims that the slow choice of player 1 is the best response of player 1 to all fast strategies $t_{2}$ of player 2 , namely, those satisfying $t_{2}<\hat{t}_{2}$, and the fast choice of player 1 is the best response of player 1 to all slow strategies $t_{2}$ of player 2 , namely, those satisfying $t_{2}>\hat{t}_{2}$; finally, both fast and slow choices of player 1 respond best to $t_{2}=\hat{t}_{2}$. We call $\hat{t}_{2}$ the switch point for player 1 .

Let us consider the function that associates to each strategy $t_{2}$ of player 2 the set of all best responses of player 1 to $t_{2}$; we call it the best response function of player 1 . The graph of the best response function of player 1 is shown in Fig. 5(a). It consists of the horizontal segment located strictly above the segment $\left(0, \hat{t}_{2}\right]$ on the $t_{2}$-axis at the level $t_{1}^{+}$, and the unbounded horizontal segment located strictly above the segment $\left[\hat{t}_{2}, \infty\right)$ on the $t_{2}$-axis at the level $t_{1}^{-}$. The points $\left(t_{1}^{+}, \hat{t}_{2}\right)$ and $\left(t_{1}^{-}, \hat{t}_{2}\right)$ lie on the graph.

A symmetric argument leads to a similar characterization of the best responses of player 1 .

Proposition 3.4. In the interval $\left(t_{2}^{-}, t_{2}^{+}\right)$, there exists a unique point $\hat{t}_{1}$ such that

$$
P_{2}\left(\hat{t}_{1}, t_{2}^{-}\right)=P_{1}\left(\hat{t}_{1}, t_{2}^{+}\right) .
$$

The set of all best responses of player 2 to $\hat{t}_{1}$ is $\left\{t_{2}, t_{2}^{+}\right\}$. If $0<t_{1}<\hat{t}_{1}$, then the unique best response of player 2 to $t_{1}$ is $t_{2}^{+}$. If $t_{1}>\hat{t}_{1}$, then the unique best response of player 2 to $t_{1}$ is $t_{2}$.

We call $t_{2}$ the fast choice of player $2, t_{2}^{+}$the slow choice of player 2 , and $\hat{t}_{2}$ the switch point for player 2 . We introduce also the best response function 


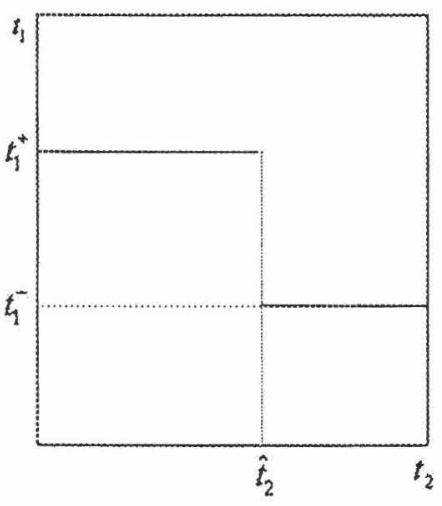

(a)

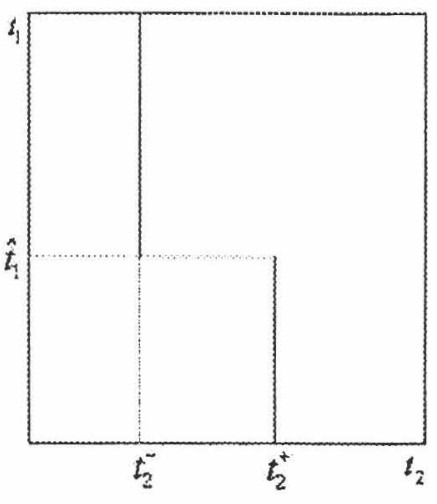

(b)

Fig. 5. (a) Best response function of player 1. (b) Best response function of player 2.

of player 2, which associates to each strategy $t_{1}$ of player 1 the set of all best responses of player 2 to $t_{1}$. The graph of the best response function of player 2 is shown in Fig. 5(b). Here, the independent variable $t_{1}$ is shown on the vertical axis and the best responses of player 2 are located on the horizontal axis. The graph of the best response function of player 2 consists of the vertical segment located to the right of the segment $\left(0, \hat{t}_{1}\right]$ on the $t_{1}$-axis at the distance $t_{2}^{+}$, and the unbounded vertical segment located to the right of the segment $\left[\hat{t}_{1}, \infty\right)$ on the $t_{1}$-axis at the distance $t_{2}$. The points $\left(\hat{t}_{1}, t_{2}^{+}\right)$and $\left(\hat{t}_{1}, t_{2}\right)$ lie on the graph.

Now, we recall the definition of a Nash equilibrium and find easily that a strategy pair $\left(t_{1}^{*}, t_{2}^{*}\right)$ is a Nash equilibrium if and only if the point $\left(t_{1}^{*}, t_{2}^{*}\right)$ belongs to the intersection of the graphs of the best response functions of players 1 and 2. Figure 5 shows that the graphs necessarily intersect. Figure 6 gives an example of the intersection.

For each intersection point [i.e., each Nash equilibrium $\left(t_{1}^{*}, t_{2}^{*}\right)$ ], point $t_{1}^{*}$ is the fast or slow choice of player 1 , and point $t_{2}^{*}$ is the fast or slow choice of player 2. In case $t_{1}^{*}$ is the fast choice of player 1 and $t_{2}^{*}$ the slow choice of player 2 , we call $\left(t_{1}^{*}, t_{2}^{*}\right)$, the fast-slow Nash equilibrium; similarly, we define the slow-fast, fast-fast, and slow-slow Nash equilibria.

Nash equilibria of different types arise under different relations between the players fast and slow choices and the switch points of their rivals. The list of all admissible cases is as follows:

$$
\begin{aligned}
& \hat{t}_{2} \geq t_{2}^{+}, \quad \hat{t}_{1}<t_{1}^{+}, \\
& \hat{t}_{2} \geq t_{2}^{+}, \quad t_{1}<\hat{t}_{1}<t_{1}^{+} \text {, }
\end{aligned}
$$




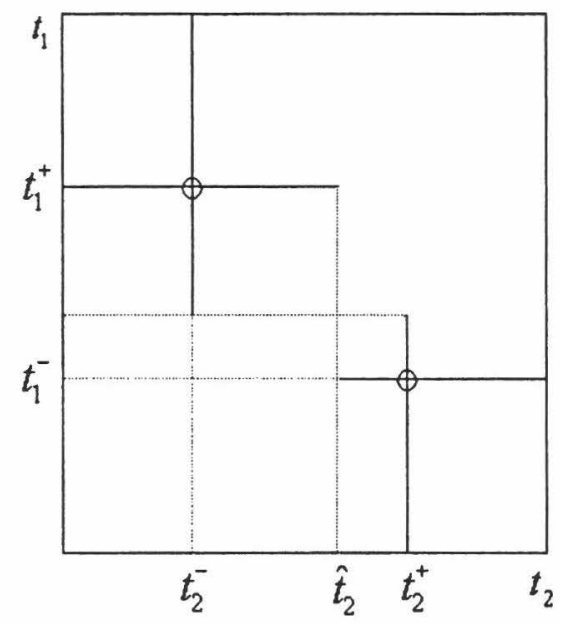

Fig. 6. Intersection of the graphs of best response functions of players 1 and 2 .

$$
\begin{array}{ll}
\hat{t}_{2} \leq t_{2}, & t_{1}<\hat{t}_{1}<t_{1}^{+}, \\
t_{2}^{-} \leq \hat{t}_{2}<t_{2}^{+}, & t_{1}^{-}<\hat{t}_{1} \leq t_{1}^{+}, \\
t_{2}<\hat{t}_{2} \leq t_{2}^{+}, & t_{1} \leq \hat{t}_{1}<t_{1}^{+}, \\
t_{2}^{-}<\hat{t}_{2}<t_{2}^{+}, & \hat{t}_{1} \leq t_{1}^{-}, \\
t_{2}<\hat{t}_{2}<t_{2}^{+}, & \hat{t}_{1} \geq t_{1}^{+}, \\
\hat{t}_{2}<t_{2}^{-}, & \hat{t}_{1} \geq t_{1}^{+} .
\end{array}
$$

An elementary analysis in the spirit of Fig. 6 leads to the full classification of the Nash equilibria in the game of timing.

Proposition 3.5. In cases (16), (17), and (21), the unique Nash equilibrium is slow-fast $\left(t_{1}^{-}, t_{2}^{+}\right)$; see Fig. 7(a), (b), (c). In cases (18), (22), and (23), the unique Nash equilibrium is fast-slow $\left(t_{1}^{+}, t_{2}^{-}\right)$; see Fig. $7(\mathrm{~d})$, (e), (f). In cases (19) and (20), the game of timing has precisely two Nash equilibria, fast-slow $\left(t_{1}, t_{2}^{+}\right)$and slow-fast $\left(t_{1}^{+}, t_{2}\right)$; see Fig. $7(\mathrm{~g})$.

Remark 3.2. Proposition 3.1 shows that the game of timing admits only fast-slow and slow-fast equilibria. 


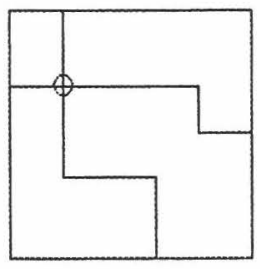

(a)

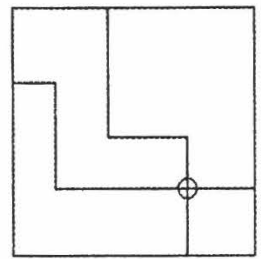

(d)

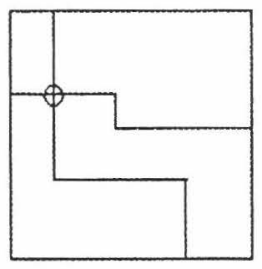

(b)

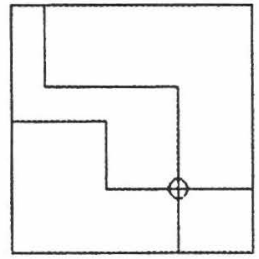

(e)

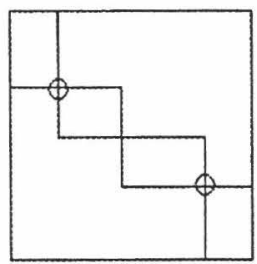

(g)

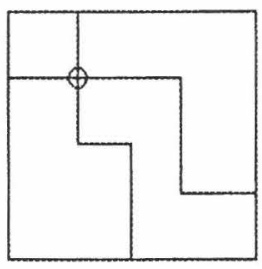

(c)

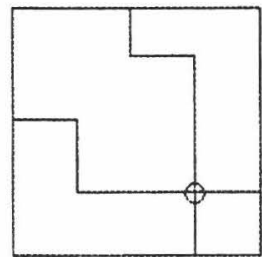

(f)

Fig. 7. (a) One equilibrium, slow-fast. (b) One equilibrium, slow-fast. (c) One equilibrium, slow-fast. (d) One equilibrium, fast-slow. (e) One-equilibrium, fast-slow. (f) One equilibrium, fast-slow. (g) Two equilibria, fast-slow and slow-fast.

Let us consider in more detail the most interesting situation where the game of timing has two Nash equilibria, fast-slow and slow-fast; i.e., (19) or (20) holds; see Fig. 7(g). By Proposition 3.1 and due to the inequalities $t_{1}^{-}<\hat{t}_{2} \leq t_{2}^{+}$, we have

$$
P_{1}\left(t_{1}^{-}, t_{2}^{+}\right) \geq P_{1}\left(t_{1}^{-}, \hat{t}_{2}\right)
$$

moreover, the inequality is strict if and only if $\hat{t}_{2}<t_{2}^{+}$. Using equality (14), Proposition 3.1 and the inequalities $t_{1}^{+}>\hat{t}_{2} \geq t_{2}$, we transform the right-hand side as follows:

$$
P_{1}\left(t_{1}^{-}, \hat{t}_{2}\right)=P_{1}\left(t_{1}^{+}, \hat{t}_{2}\right)=P_{1}\left(t_{1}^{+}, t_{2}^{-}\right) .
$$

Thus, for the fast-slow and slow-fast equilibria $\left(t_{1}, t_{2}^{+}\right)$and $\left(t_{1}^{+}, t_{2}\right)$, we have

$$
P_{1}\left(t_{1}^{-}, t_{2}^{+}\right) \geq P_{1}\left(t_{1}^{+}, t_{2}\right) \text {. }
$$


Moreover, the inequality is strict if $t_{2}<t_{2}^{+}$. If this is so, player 1 prefers the fast-slow equilibrium; otherwise, the fast-slow and slow-fast equilibria are equivalent for this player. Similarly, we state that, if $\hat{t}_{1}<t_{1}^{+}$, player 2 prefers the slow-fast equilibrium; otherwise, the equilibria are equivalent for this player. Thus, generally, each player prefers his fast equilibrium.

Let us give an exact formulation.

Proposition 3.6. Let the game of timing have two Nash equilibria; i.e., let (19) or (20) hold. Then:

(i) $P_{1}\left(t_{1}^{-}, t_{2}^{+}\right) \geq P_{1}\left(t_{1}^{+}, t_{2}^{-}\right)$; moreover, the inequality is strict if and only if $\hat{t}_{2}<t_{2}^{+}$

(ii) $P_{2}\left(t_{1}, i_{2}^{+}\right) \geq P_{2}\left(t_{1}^{+}, i_{2}\right)$; moreover, the inequality is strict if and only if $\hat{t}_{1}<t_{1}^{+}$.

Remark 3.3. Let the game have two equilibria [i.e., (19) or (20) hold]. Assume that the fast-slow and slow-fast equilibria are equivalent to player 1, i.e.,

$$
P_{1}\left(t_{1}^{-}, t_{2}^{+}\right)=P_{1}\left(t_{1}^{+}, t_{2}^{-}\right)
$$

Then, by Proposition 3.6(i), $\hat{t}_{2} \geq t_{2}^{+}$. As (19), (20) show, we actually have $\hat{t}_{2}=t_{2}^{+}$, which is an exceptional situation for the case of two equilibria. Hence,

$$
\hat{t}_{1}<t_{2}^{+} \leq \hat{t}_{2}<t_{1}^{+} \text {. }
$$

By Proposition 3.6(ii),

$$
P_{2}\left(t_{1}, t_{2}^{+}\right)>P_{2}\left(t_{1}^{+}, t_{2}^{-}\right)
$$

In other words, the slow-fast equilibrium is strictly preferable for player 2. In the symmetric case, where the fast-slow and slow-fast equilibria are equivalent to player 2 , i.e.,

$$
P_{1}\left(t_{1}, t_{2}^{+}\right)=P_{1}\left(t_{1}^{+}, t_{2}\right)
$$

we find similarly that the fast-slow equilibrium is strictly preferable for player 1 . Thus, in those exceptional cases where one of the players has no preference in choosing an equilibrium, the other player strictly prefers his fast equilibrium. 
Remark 3.4. Let us assume that the parameters of projects 1 and 2 are identical; i.e.,

$$
C_{1}(t)=C_{2}(t) \text { and } \quad B_{1}(t, s)=B_{2}(s, t), \quad \text { for all positive } t \text { and } s .
$$

Then, the game of timing takes a symmetric form. The players have the same fast and slow choices and switch times,

$$
t_{1}=t_{2}, \quad t_{1}^{+}=t_{2}^{+}, \quad \hat{t}_{2}=\hat{t}_{1},
$$

Hence, (19) and (20) hold. By Proposition 3.5, the game of timing has the fast-slow and slow-fast equilibria. The inequality $\hat{t}_{2}<t_{2}^{+}$is equivalent to $\hat{t}_{2}<t_{1}^{+}$, which holds trivially [see (6)]. By Proposition 3.6, we conclude that

$$
P_{1}\left(t_{1}^{+}, t_{2}^{+}\right)>P_{1}\left(t_{1}^{+}, t_{2}^{-}\right)
$$

Similarly, we find that

$$
P_{2}\left(t_{1}, t_{2}^{+}\right)>P_{2}\left(t_{1}^{+}, t_{2}\right)
$$

Thus, in the symmetric game of timing, player 1 prefers the fast-slow equilibrium and player 2 prefers the slow-fast equilibrium. Obviously, the situation does not change if the parameters of projects 1 and 2 are sufficiently close to each other. The question of a practical choice of an equilibrium in the case where the players have different preferences arises. Here, we do not argue on this; we note only that game theory does not provide any clear recommendations in this respect.

\section{Solution Algorithm}

For convenience, we represent the obtained classification of the Nash equilibria in table form (see Table 1).

We conclude the general part of our study with the description of an algorithm that finds the Nash equilibria in the game of timing. The algorithm refers to the definitions of the players fast and slow choices $t_{i}^{-}$and $t_{i}^{+}, i=1,2$, the players switch times $\hat{t}_{i}, i=1,2$, and Table 1 .

Step 1. Use definitions (4) and (5) for finding the players fast and slow choices $t_{i}$, and $t_{2}^{+}, i=1,2$.

Step 2. Use definitions (14) and (15) for finding the players switch times $\hat{t}_{i}, i=1,2$.

Step 3. Use Table 1 for identifying the Nash equilibria. 
Table 1. Classification of Nash equilibria in the game of timing (table form of Proposition 3.5).

\begin{tabular}{|c|c|c|c|}
\hline Case & $\begin{array}{l}\text { Number of } \\
\text { equilibria }\end{array}$ & $\begin{array}{l}\text { Types of } \\
\text { equilibria }\end{array}$ & Notation \\
\hline $\begin{array}{l}\hat{t}_{1}<t_{1}^{-} \\
\hat{t}_{2} \geq t_{2}^{+}\end{array}$ & 1 & slow-fast & $\left(t_{1}^{+}, t_{2}\right)$ \\
\hline $\begin{array}{l}f_{1}<\hat{t}_{1}<t_{1}^{+} \\
\hat{f}_{2} \geq t_{2}^{+}\end{array}$ & 1 & slow-fast & $\left(i_{1}^{+}, t_{2}\right)$ \\
\hline $\begin{array}{l}t_{1}<\hat{t}_{1}<t_{1}^{+} \\
\hat{t}_{2} \leq t_{2}\end{array}$ & 1 & fast-slow & $\left(t_{1}, t_{2}^{+}\right)$ \\
\hline $\begin{array}{l}t_{1}^{-}<\hat{t}_{1} \leqslant t_{1}^{+} \\
t_{2}^{-} \leqslant \hat{t}_{2}<t_{2}^{+}\end{array}$ & 2 & $\begin{array}{l}\text { fast-slow } \\
\text { slow-fast }\end{array}$ & $\begin{array}{l}\left(t_{1}^{-}, t_{2}^{*}\right) \\
\left(t_{1}^{+}, t_{2}^{-}\right)\end{array}$ \\
\hline $\begin{array}{l}t_{1} \leq \hat{t}_{1}<t_{1}^{*} \\
t_{2}^{-}<\hat{t}_{2} \leq t_{2}^{+}\end{array}$ & 2 & $\begin{array}{l}\text { fast-slow } \\
\text { slow-fast }\end{array}$ & $\begin{array}{l}\left(t_{1}^{-}, t_{2}^{+}\right) \\
\left(t_{1}^{+}, t_{2}\right)\end{array}$ \\
\hline $\begin{array}{l}\hat{t}_{1} \leq t_{1} \\
t_{2}<\hat{t}_{2}<t_{2}^{+}\end{array}$ & 1 & slow-fast & $\left(t_{1}^{+}, t_{2}\right)$ \\
\hline $\begin{array}{l}\hat{t}_{1} \geqslant t_{1}^{+} \\
t_{2}<\hat{t}_{2}<t_{2}^{+}\end{array}$ & 1 & fast-slow & $\left(t_{1}, t_{2}^{+}\right)$ \\
\hline $\begin{array}{l}\hat{t}_{1} \geq t_{1}^{+} \\
\hat{t}_{2}<t_{2}^{-}\end{array}$ & 1 & fast-slow & $\left(t_{1}^{-}, l_{2}^{+}\right)$ \\
\hline
\end{tabular}

\section{Gas Pipeline Game}

In this section, we apply the suggested solution method to a model described in Ref. 1. Wishing to demonstrate a clear analytic result, we consider a simplified version of the model. Namely, we eliminate the price of liquid natural gas, which acts as an upper bound for the price of gas in the original model; we do not introduce the upper bounds for the rates of supply or the pipelines capacities; we assume that the costs for extraction and transportation of gas are functions of time only; finally, we analyze the competition of two pipeline projects (as our theory prescribes).

The model is as follows. The cost $C_{i}\left(t_{i}\right)$ for finalizing the construction of pipeline $i, i=1,2$, at time $t_{i}$ is defined to be the minimum of the integral investment

$$
I_{i}\left(r_{i}\right)=\int_{0}^{t_{i}} e^{-\lambda t} r_{i}(t) d t .
$$

Here, $\lambda$ is a positive discount. The minimum is taken over all admissible open-loop investment strategies $r_{i}(t)$ of player $i$. An admissible open-loop investment strategy of player $i$ (for a commercialization time $t_{i}$ ) is modeled as an integrable control function,

$$
r_{i}(t)>0 \text {, }
$$


that brings the accumulated investment $x_{i}(t)$ from 0 to the prescribed commercialization level $\tilde{x}_{i}>0$ at time $t_{i}$. Thus, for the initial and final values of the accumulated investment, we have

$$
x_{i}(0)=0, \quad x_{i}\left(t_{i}\right)=\bar{x}_{i} .
$$

The dynamics of $x_{i}(t)$ is modeled as

$$
\dot{x}_{i}(t)=-\sigma x_{i}(t)+r_{i}^{\gamma}(t) .
$$

Here, $\sigma$ is a positive obsolescence coefficient and $\gamma$ is a delay parameter, located strictly between 0 and 1 . In the terminology of control theory (see Ref. 15), the cost $C_{i}\left(t_{i}\right)$ is defined to be the optimal value in the problem of minimizing the performance index $I_{i}\left(r_{i}\right)$ for the control system (26), (24), subject to the boundary constraints $(25)$.

The upper and lower benefit rates $b_{i 1}(t)$ and $b_{i 2}(t)$ for player $i$ at time $t>0$ are found as equilibrium payoffs in the static supply game modeling the instantaneous gas market. In the supply game arising at time $t$, the strategies $y_{i}$ of player $i$ are nonnegative rates of supply and the payoff to player $i$ is defined as

$$
p_{i}\left(y_{1}, y_{2} \mid t\right)=e^{-\lambda t}\left[\pi(t, y)-c_{i}(t)\right] y_{i} .
$$

Here, $y$ is the total rate of supply, $\pi(t, y)$ is the price of gas, and $c_{i}(t)>0$ is the cost for extraction and transportation of gas for player $i$. The price of gas is modeled as

$$
\pi(y \mid t)=(g(t) / y)^{\beta},
$$

where $g(t)>0$ is the consumer GDP at time $t$ and $\beta$ is the inverse to the price elasticity of gas demand; we have

$$
0<\beta<1 \text {. }
$$

The total supply $y$ equals $y_{i}$ if player $i$ occupies the market solely and equals $y_{1}+y_{2}$ if both players operate on market.

The next proposition gives the expressions for the costs $C_{i}\left(t_{i}\right)$, rates of cost reduction $a_{i}\left(t_{i}\right)$, and upper and lower benefit rates $b_{i 1}\left(t_{i}\right)$ and $b_{i 2}\left(t_{i}\right)$, $i=1,2$. We need the following assumption.

Assumption 5.1. It holds that

$$
1-(2-\beta) c_{i}(t) /\left[c_{1}(t)+c_{2}(t)\right]>0, \quad i=1,2 .
$$

Remark 5.1. Condition (28) implies that the costs $c_{1}(t)$ and $c_{2}(t)$ are relatively close to each other. Indeed, in the extremal case where $c_{1}(t)=$ $c_{2}(t)=c(t), \quad(28)$ is equivalent to the trivial inequality $\beta>0$. Another 
interpretation of condition (28) is that $\beta$ is close to 1 . Indeed, in the limiting case where $\beta=1,(28)$ is equivalent to the trivial inequality

$$
1-c_{i}(t) /\left[c_{1}(t)+c_{2}(t)\right]>0 \text {. }
$$

Proposition 5.1. For player $i, i=1,2$, the following formulas hold:

(a) The cost $C_{i}\left(t_{i}\right)$ is given by

$$
C_{i}\left(t_{i}\right)=\rho^{\alpha-1} e^{-\lambda t_{i}} \bar{x}_{i}^{\alpha} /\left(1-e^{-\rho t_{i}}\right)^{\alpha-1},
$$

where

$\alpha=1 / \gamma, \quad \rho=(\alpha \sigma+\lambda) /(\alpha-1)$.

(b) The rate of cost reduction $a_{i}\left(t_{i}\right)$ is given by

$a_{i}(t)=\rho^{\alpha-1} \bar{x}_{i}^{\alpha} e^{-\lambda t}\left(\lambda+v e^{-\rho t}\right) /\left(1-e^{-\rho t}\right)^{\alpha}$,

where

$v=\alpha \sigma$.

(c) The upper benefit rate $b_{i 1}\left(t_{i}\right)$ is given by

$b_{i 1}(t)=e^{-\lambda t}(1-\beta)^{1 / \beta-1} g(t) / c_{i}^{1 / \beta-1}(t)$.

(d) If Assumption 5.1 holds, the lower benefit rate $b_{i 2}\left(t_{i}\right)$ is given by

$$
b_{i 2}(t)=e^{-\lambda t}(2-\beta)^{1 / \beta \cdots 1}\left\{1-\frac{(2-\beta) c_{i}(t)}{\left[c_{1}(t)+c_{2}(t)\right]}\right\}^{2} g(t) /\left[c_{1}(t)+c_{2}(t)\right]^{1 / \beta-1} .
$$

(e) Under Assumption 5.1, the following inequality is valid:

$b_{i 1}(t)>b_{i 2}(t)$;

see (2) and (3).

In what follows, we assume that $c_{i}(t), i=1,2$, and $g(t)$ are defined on the positive half axis and are continuous. We also fix the functions described in Proposition 5.1 and introduce the next assumption.

Assumption 5.2. For $i=1,2$, the functions

$$
\begin{aligned}
& h_{i 1}(t)=g(t) / c_{i}(t)^{1 / \beta-1}, \\
& h_{i 2}(t)=\left\{1 \cdots \frac{(2-\beta) c_{i}(t)}{\left[c_{1}(t)+c_{2}(t)\right]}\right\}^{2} g(t) /\left[c_{1}(t)+c_{2}(t)\right]^{1 / \beta-1},
\end{aligned}
$$


$t>0$, increase and tend to infinity as $t$ tends to infinity, and the integral $\int_{0}^{\infty} e^{-\lambda t} h_{i 1}(t) d t$ is finite.

Remark 5.2. Assumption 5.2 holds if $g(t)$ [the consumer GDP] and the costs $c_{i}(t)$ grow exponentially,

$$
g(t)=g^{0} e^{\zeta_{t}}, \quad c_{i}(t)=c_{i}^{0} e^{\omega t}, \quad i=1,2,
$$

$\zeta$ and $\omega$ are nonnegative, and

$$
0<\kappa<\lambda,
$$

where

$$
\kappa=\zeta \cdots(1 / \beta \cdots 1) \omega .
$$

Note that $g_{0}$ is the consumer GDP at time 0 and $c_{i}^{0}$ is the cost for transportation and extraction for player $i$ at time 0 .

The theory described earlier for the general case is applicable for the model considered. Namely, the following is true.

Proposition 5.2. Let Assumptions 5.1 and 5.2 hold. Then, Assumptions 2.1 and 2.2 hold. Moreover, the fast choice $t_{i}$ of player $i, i=1,2$, is the unique solution of the algebraic equation

$$
\rho^{\alpha-1} \bar{x}_{i}^{\alpha} /(1-\beta)^{1 / \beta-1}=\left[\left(1 \cdots e^{-\rho t}\right)^{\alpha} /\left(\lambda+v e^{-\rho t}\right)\right] h_{i 1}(t) ;
$$

the slow choice $t_{i}^{+}$of player $i$ is the unique solution of the algebraic equation

$$
\rho^{\alpha-1} \bar{x}_{i}^{\alpha} /(2-\beta)^{1 / \beta-1}=\left[\left(1-e^{-\rho t}\right)^{\alpha} /\left(\lambda+v e^{-\rho t}\right)\right] h_{i 2}(t) .
$$

Thus, under Assumptions 5.1 and 5.2, the general algorithm for the resolution of the game of timing (see Section 4) is specified as follows.

Step 1. Solve equations (40) and (41) for finding the players fast and slow choices $t_{i}$ and $t_{2}^{+}$, respectively, $i=1,2$.

Step 2. Use equalities (14) and (15) for finding the players switch times $\hat{t}_{i}, i=1,2$.

Step 3. Use Table 1 for identifying the Nash equilibria in the game of timing.

As a specific example, let us consider the case described in Remark 5.2. Thus, in what follows, we assume that $g(t)$ and $c_{i}(t), i=1,2$, are given by (37) and inequality (38) is satisfied. Formulas (33) and (34) for $b_{i 1}(t)$ and $b_{i 2}(t)$ are 
specified as

$$
b_{i 1}(t)=b_{i 1}^{0} e^{-\psi t}, \quad b_{i 2}(t)=b_{i 2}^{0} e^{-\psi t},
$$

where

$$
\begin{aligned}
& \psi=\lambda-\kappa, \\
& b_{i 1}^{0}=(1-\beta)^{1 / \beta-1} g^{0} /\left(c_{i}^{0}\right)^{1 / \beta-1}, \\
& b_{i 2}^{0}=(2-\beta)^{1 / \beta-1}\left[1-(2-\beta) c_{i}^{0} /\left(c_{1}^{0}+c_{i}^{0}\right)\right]^{2} g^{0} /\left(c_{1}^{0}+c_{2}^{0}\right)^{1 / \beta-1} .
\end{aligned}
$$

Using the definition of the total benefit $B_{i}\left(t_{1}, t_{2}\right)$ of player $i$ [see (8) and (9)] and the expression (29) for the cost $C_{i}\left(t_{i}\right)$, we find an explicit formula for the total profit $P_{i}\left(t_{1}, t_{2}\right)$ [see $(10)$ ] of player $i$, which is determined by the player strategies $t_{1}$ and $t_{2}$. We have

$$
\begin{aligned}
P_{1}\left(t_{1}, t_{2}\right)= & -\rho^{\alpha-1} e^{-\lambda t_{1}} \bar{x}_{1}^{\alpha} /\left(1-e^{-\rho t_{1}}\right)^{\alpha-1} \\
& + \begin{cases}b_{11}^{0} e^{-\psi t_{1}} / \psi+\left(b_{12}^{0}-b_{11}^{0}\right) e^{-\psi t_{2}} / \psi, & \text { if } t_{1} \leq t_{2}, \\
b_{12}^{0} e^{-\psi t_{1}} / \psi, & \text { if } t_{1} \geq t_{2},\end{cases} \\
P_{2}\left(t_{1}, t_{2}\right)= & -\rho^{\alpha-1} e^{-\lambda t_{2}} \bar{x}_{2}^{\alpha} /\left(1-e^{-\rho t_{2}}\right)^{\alpha-1} \\
& + \begin{cases}b_{21}^{0} e^{-\psi t_{1}} / \psi+\left(b_{22}^{0}-b_{21}^{0}\right) e^{-\psi t_{1}} / \psi, & \text { if } t_{2} \leq t_{1}, \\
b_{22}^{0} e^{-\psi t_{2}} / \psi, & \text { if } t_{2} \geq t_{1} .\end{cases}
\end{aligned}
$$

Figure 8 shows the Maple-simulated landscape of $P_{1}\left(t_{1}, t_{2}\right)$ for

$$
\begin{array}{ll}
\alpha=1.5, & \lambda=0.3, \quad \sigma=0.3, \quad g^{0}=3.5, \quad \bar{x}_{1}=0.7, \\
\beta=0.5, \quad c_{1}^{0}=c_{2}^{0}=0.2 .
\end{array}
$$

Recall that, by Proposition 3.3, the critical points $\hat{t}_{2}$ and $\hat{t}_{1}$ needed for the identification of the type of the equilibria in the game of timing (see Table 1) are found from the equalities

$$
P_{1}\left(t_{1}^{-}, \hat{t}_{2}\right)=P_{1}\left(t_{1}^{+}, \hat{t}_{2}\right) \text { and } P_{1}\left(\hat{t}_{1}, t_{2}^{-}\right)=P_{1}\left(\hat{t}_{1}, t_{2}^{+}\right) \text {, }
$$

respectively. In the situation considered now, the critical points are given explicitly. The next proposition is true.

Proposition 5.3. For $i=1,2$, we have

$$
\hat{t}_{i}=-(1 / \psi) \log \left[\psi G_{i} /\left(b_{i 2}^{0}-b_{i 1}^{0}\right)\right]
$$




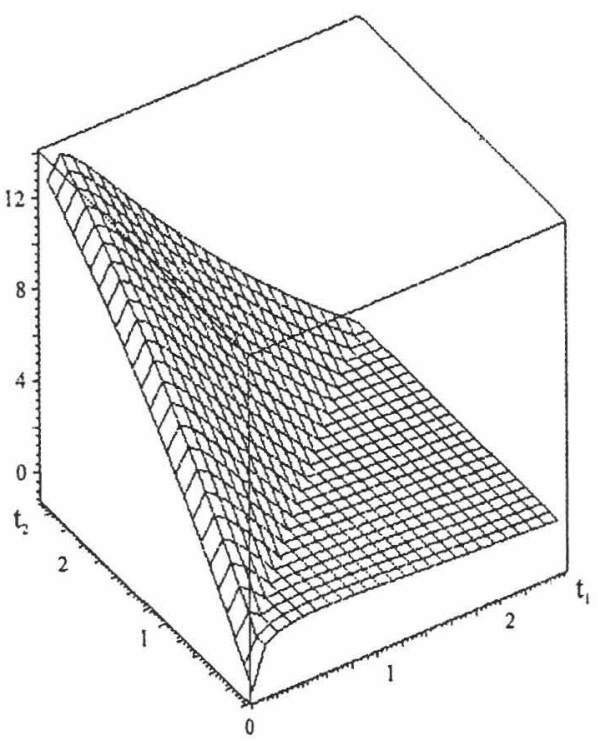

(a)

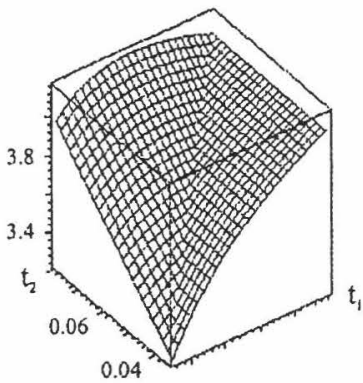

(b)

Fig. 8. Payoff landscape for player 1: (a) for large $t_{2}$, the fast choice $t_{1}^{-}$replies best; (a) for small $t_{2}$, the slow choice $t_{1}^{+}$replies best.

where

$$
\begin{aligned}
& G_{i}=-\rho^{\alpha-1} e^{-\lambda t_{i}^{+}} \bar{x}_{1}^{\alpha} /\left(1-e^{-\rho t_{1}^{+}}\right)^{\alpha-1}+b_{i 2}^{0} e^{-\psi t_{1}^{+}} / \psi \\
& +\rho^{\alpha-1} e^{\lambda r_{i}^{-}} \bar{x}_{1}^{\alpha} /\left(1-e^{-\rho r_{i}^{-}}\right)^{\alpha-1}-b_{i 1}^{0} e^{-\psi r_{i}} / \psi \text {. }
\end{aligned}
$$

The next proposition specifies Proposition 5.2.

Proposition 5.4. Let $g(t)$ and $c_{i}(t), i=1,2$, be given by (37) and let inequality (38) be satisfied. Then, for every player $i, i=1,2$, the following assertions hold.

(a) The fast choice $t_{i}$ of player $i$ is the unique solution of the algebraic equation

$l_{i} w_{i}=\epsilon^{k \cdot t}\left(1-e^{-\rho \prime}\right)^{\alpha} /\left(\lambda+v \epsilon^{-\rho \prime}\right)$,

where

$$
\begin{aligned}
& l_{i}=\rho^{\alpha-1} /(1-\beta)^{1 / \beta-1} g^{0}, \\
& w_{i}=\bar{x}_{i}^{\alpha}\left(c_{i}^{0}\right)^{1 / \beta-1} .
\end{aligned}
$$


(b) The slow choice $t_{i}^{+}$of player $i$ is the unique solution of the algebraic equation

$l_{i} z_{i}=e^{\kappa t}\left(1 \cdots e^{-\rho t}\right)^{\alpha} /\left(\lambda+v e^{-\rho t}\right)$,

where $l_{i}$ is defined by (47) and

$z_{i}=\bar{x}_{i}^{\alpha}\left(c_{1}^{0}+c_{2}^{0}\right)^{1 / \beta-1} /\left[1-(2-\beta) c_{i}^{0} /\left(c_{1}^{0}+c_{2}^{0}\right)\right]^{2}$.

Thus, under the assumptions of Remark 5.2, the suggested solution algorithm for the game of timing (Section 4) takes the following form.

Step 1. Solve equations (46) and (49) for finding the players fast and slow choices $t_{i}^{-}$and $t_{i}^{+}$, respectively, $i=1,2$.

Step 2. Use formula (44) for finding the players switch times $\hat{t}_{i}, i=1,2$.

Step 3. Use Table 1 for identifying the Nash equilibria in the game of timing.

Figure 9 shows the Maple-simulated graphs of the fast choice $t_{1}^{-}$and slow choice $t_{1}^{+}$of player 1 as functions of $\bar{x}_{1}$ and $c_{1}^{0}=c_{2}^{0}$ for different values of $\beta$ and

$$
\alpha=1.5, \quad \lambda=0.3, \quad \sigma=0.3, \quad g^{0}=3.5 .
$$

\section{Case Study}

In this section, we describe the game of timing in application to the Caspian and China gas markets. The values of the model parameters are

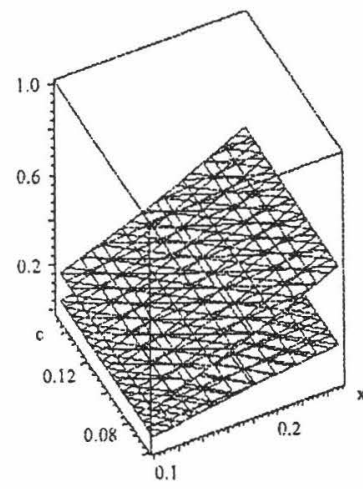

(a)

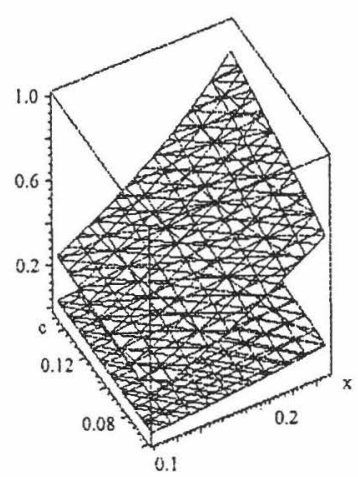

(b)

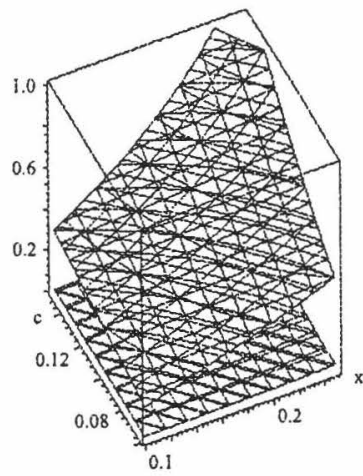

(c)

Fig. 9. Graphs of the fast choice $t_{1}$ and slow choice $t_{i}^{+}$of player 1 as functions of $\bar{x}_{1}$ and $c_{1}$ : (a) $\beta=0.97$; (b) $\beta=0.75$; (c) $\beta=0.5$. 
based on preliminary expert estimates. Our first case study deals with the competition of two major gas pipeline projects in the Caspian region, the Blue Stream Project of the Russian GAzProm Company (project 1), which is aimed at delivering Russian gas to Turkey under the Black Sea; and the Trans-Caspian Project (project 2) directed from Turkmenistan underneath the Caspian Sea through Azerbaijan and Georgia to Turkey. In this case study, the parameters of the model are chosen as follows: discount rate $\lambda=0.1$; obsolescence coefficient $\sigma=0.3$; delay coefficient, $\gamma=0.65$; inverse to the price elasticity of gas demand $\beta=0.55$; initial level of the consumer GDP $g^{0}=214.6$; growth rate of the consumer GDP $\zeta=0.1$; growth rate of the extraction costs $\omega=0.15$; initial extraction costs $c_{1}^{0}=67.3, c_{2}^{0}=78.4$; commercialization levels of the accumulated investments $\bar{x}_{1}=4.0, \bar{x}_{2}=2.5$. It is assumed that the projects start in 2001 .

For these parameters, there exist two Nash equilibria in the game of timing, the fast-slow equilibrium

$$
\left(t_{1}, t_{2}^{+}\right)=(2002.8,2005.2)
$$

and the slow-fast equilibrium

$$
\left(t_{1}^{+}, t_{2}\right)=(2004.6,2002.2) \text {. }
$$

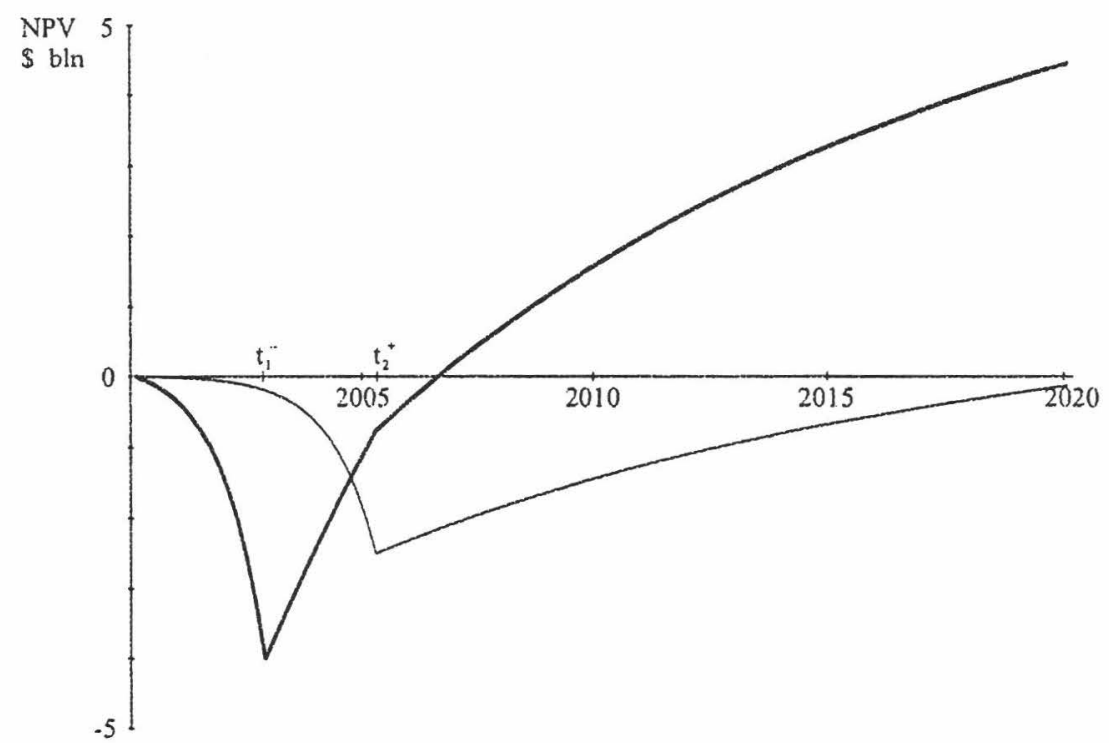

Fig. 10. NPV dynamics for the fast-slow scenario of the gas pipeline competition in the Caspian region. 
Figures 10 and 11 depict the dynamics of the net present values NPV, $P_{i}=P_{i}\left(t, t_{1}, t_{2}\right)$ (in billion dollars)

$$
P_{i}\left(t, t_{1}, t_{2}\right)= \begin{cases}-C_{i}(t), & \text { if } 0 \leq t<t_{i}, \\ -C_{i}\left(t_{i}\right)+\int_{t_{i}}^{t} b_{i}\left(\tau \mid t_{j}\right) d \tau, & \text { if } t \geq t_{i},\end{cases}
$$

with $i, j=1,2, i \neq j$, for project 1 (Blue Stream) and project 2 (Trans-Caspian) under the fast-slow Nash equilibrium investment scenario $\left(t_{1}, t_{2}\right)=\left(t_{1}^{-}, t_{2}^{+}\right)$ and the slow-fast Nash equilibrium investment scenario $\left(t_{1}, t_{2}\right)=\left(t_{1}^{+}, t_{2}^{-}\right)$, respectively. The heavy line and the fine line show the NPV dynamics of Blue Stream and Trans-Caspian, respectively.

Our second case study is related to the planned projects of gas pipelines from Russia to China. Two potential competitors on the North China gas market are the Kovikta-Zabaikalsk-Kharbin pipeline (project 1) stretched from the Irkutsk region to North China, and the Sakhalin-KhabarovskKharbin pipeline (project 2). The following values of the model parameters are chosen:

$$
\begin{aligned}
& \lambda=0.1, \quad \sigma=0.3, \quad \gamma=0.58, \quad \beta=0.46, \quad g^{0}=1157, \\
& \zeta=0.0668, \quad \omega=0.05, \quad c_{1}^{0}=57, \quad c_{2}^{0}=68, \quad \bar{x}_{1}=6, \quad \bar{x}_{2}=3 \text {. }
\end{aligned}
$$

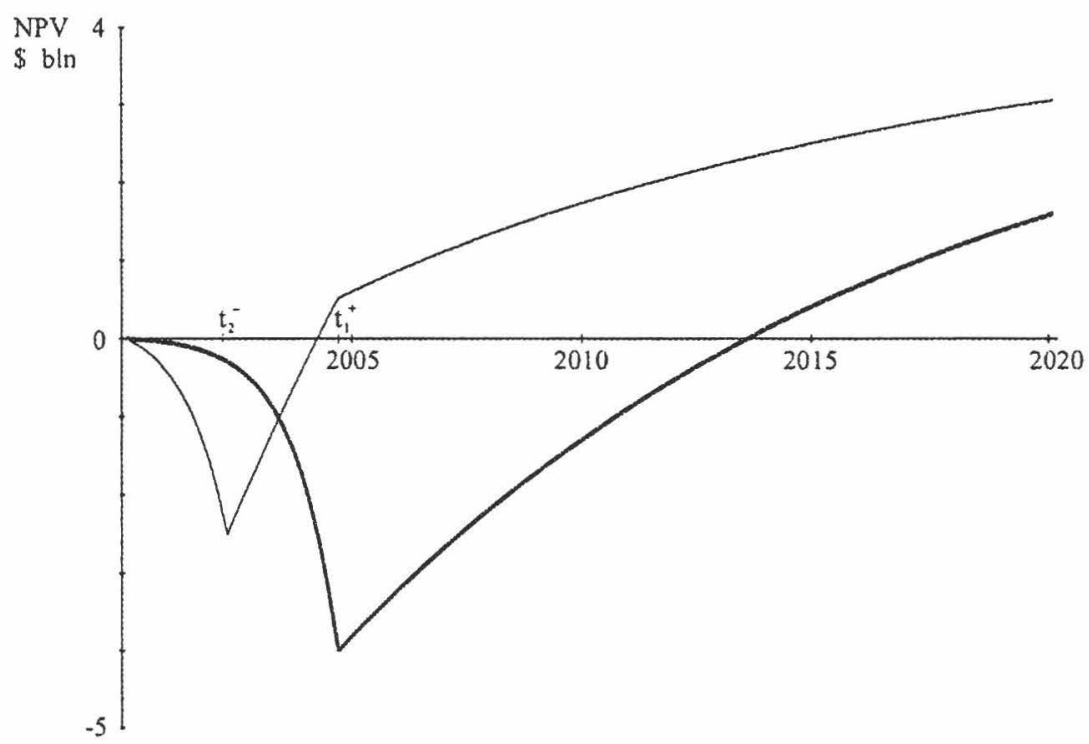

Fig. 11. NPV dynamics for the slow-fast scenario of the gas pipeline competition in the Caspian region. 


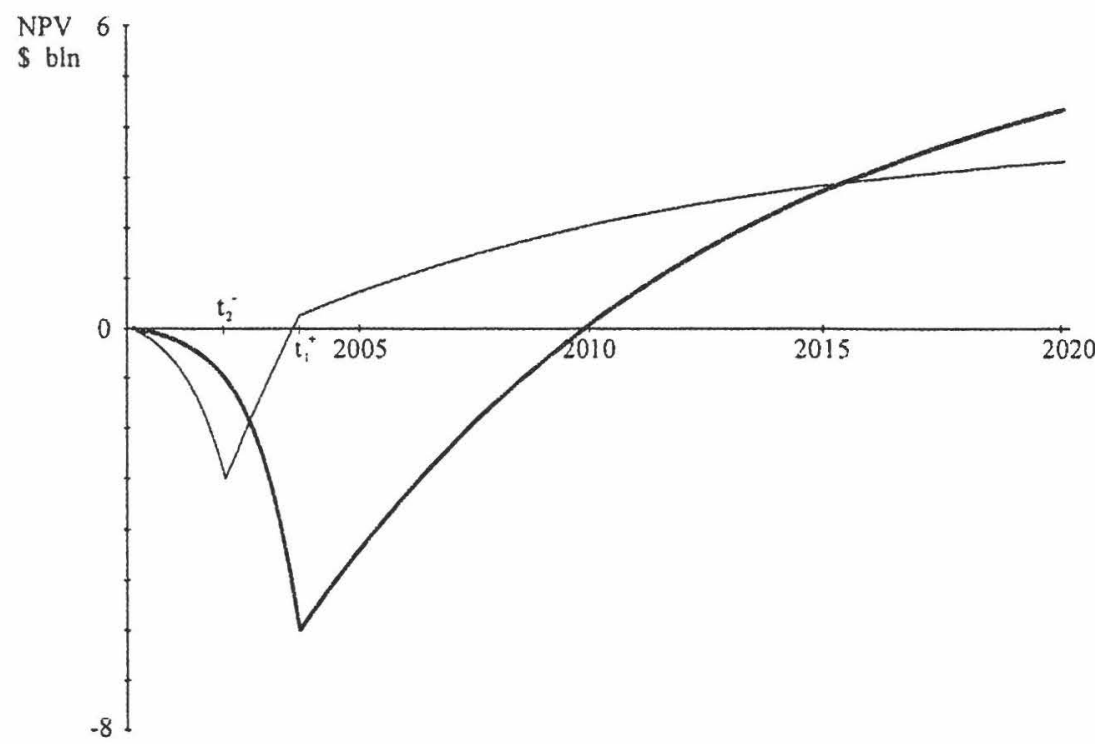

Fig. 12. NPV dynamics for the Nash equilibrium scenario of the planned pipeline projects to the gas market in China.

The initial year for the projects is set in 2001 .

In this case study, there exists a unique slow-fast Nash equilibrium

$$
\left(t_{1}^{+}, t_{2}^{-}\right)=(2003.6,2002)
$$

Figure 12 shows the dynamics of the NPV (51), $P_{i}=P_{i}\left(t, t_{1}, t_{2}\right), i=1,2$, for projects 1 and 2 under the slow-fast Nash equilibrium investment scenario $\left(t_{1}, t_{2}\right)=\left(t_{1}^{+}, t_{2}^{-}\right)$. The heavy line and the fine line depict the NPV dynamics of the Kovikta-Zabaikalsk-Kharbin project and the Sakhalin-KhabarovskKharbin project, respectively.

The results demonstrated on Figs. 10-12 have been calculated using the G-TIME software package elaborated and tested by O. I. Nikonov and Y. V. Minoullin.

\section{Appendix: Proof of the Main Results}

Here, we prove Propositions 5.1 to 5.4 .

\section{Proof of Proposition 5.1.}

Step 1. Formula (29) was obtained in Ref. 14. 
Step 2. The differentiation of (29) gives

$$
\begin{aligned}
C_{i}^{\prime}\left(t_{i}\right) & =\rho^{\alpha-1} \frac{\lambda e^{-\lambda t_{i}} \tilde{x}_{i}^{\alpha}\left(1-e^{-\rho t_{i}}\right)^{\alpha-1}-e^{-\lambda t_{i}} \tilde{x}_{i}^{\alpha}(\alpha-1)\left(1-e^{-\rho t_{i}}\right)^{\alpha-2} \rho e^{-\rho t_{i}}}{\left(1-e^{-\rho t_{i}}\right)^{2 \alpha-2}} \\
& =\frac{\rho^{\alpha-1} e^{-\lambda t_{i}} \bar{x}_{i}^{\alpha}\left(1-e^{-\rho t_{i}}\right)^{\alpha-2}}{\left(1-e^{-\rho t_{i}}\right)^{2 \alpha-2}}\left[-\lambda\left(1-e^{-\rho t_{i}}\right) \cdots(\alpha-1) \rho e^{-\rho t_{i}}\right] \\
& =-\rho^{\alpha-1} \bar{x}_{i}^{\alpha} \frac{e^{-\lambda t_{i}}}{\left(1-e^{-\rho t_{i}}\right)^{\alpha}}\left[\lambda\left(1-e^{-\rho t_{i}}\right)+(\alpha-1) \rho e^{-\rho t_{i}}\right] \\
& =-\rho^{\alpha-1} \frac{e^{-\lambda t_{i}} \bar{x}_{i}^{\alpha}}{\left(1-e^{-\rho t_{i}}\right)^{\alpha}}\left[\lambda+(\rho(\alpha-1)-\lambda) e^{-\rho t_{i}}\right] \\
& =-\rho^{\alpha-1} \frac{e^{-\lambda t_{i}} \tilde{x}_{i}^{\alpha}\left(\lambda+v e^{-\rho t_{i}}\right)}{\left(1-e^{-\rho t_{i}}\right)^{\alpha}} .
\end{aligned}
$$

For the last transformation, we have used the equality

$$
\rho(\alpha-1)-\lambda=\alpha \sigma
$$

following from (30) and the notation (32). For $a_{i}\left(t_{i}\right)=-C_{i}^{\prime}\left(t_{i}\right)$ [see (1)], we have (31).

Step 3. Assume that player $i$ occupies the market solely. Then, the price is given by

$$
\pi(y \mid t)=\left(g(t) / y_{i}\right)^{\beta},
$$

and the payoff $p_{i}\left(y_{1}, y_{2} \mid t\right)$ to player $i$ equals

$$
p_{i}\left(y_{i} \mid t\right)=e^{-\lambda_{i}}\left[g(t)^{\beta} y_{i}^{1 \cdots \beta} \cdots c_{i}(t) y_{i}\right]
$$

The supply game is reduced to an optimization problem, and $b_{i 1}(t)$ is found as the maximum of $p_{i}\left(y_{i} \mid t\right)$ over all positive $y_{i}$. Since $p_{i}\left(y_{i} \mid t\right)$ is strictly concave in $y_{i}$, its maximum is reached at the unique point $y_{i}(t)>0$ such that

$$
d p_{i}\left(y_{i}(t) \mid t\right) / d y_{i}=e^{\cdots \lambda} g(t)^{\beta}\left[(1 \cdots \beta) y_{i}^{-\beta}(t) \cdots c_{i}(t)\right]=0 .
$$

Hence,

$$
y_{i}(t)=\left[g(t) / c_{i}(t)^{1 / \beta}\right](1 \cdots \beta)^{1 / \beta} .
$$

Recall that

$$
b_{i 1}(t)=p_{i}\left(y_{i}(t) \mid t\right)
$$

and substitute $y_{i}=y_{i}(t)$ into (53). We get

$$
\begin{aligned}
b_{i 1}(t) & =e^{-\lambda t}\left[g^{\beta}(t) / y_{i}^{\beta}-c_{i}(t)\right] y_{i}(t) \\
& =e^{-\lambda t}\left[c_{i}(t) /(1 \cdots \beta)-c_{i}(t)\right]\left[g(t) / c_{i}(t)^{1 / \beta}\right](1 \cdots \beta)^{1 / \beta},
\end{aligned}
$$


and finally,

$$
b_{i 1}(t)=e^{-\lambda t} \beta(1-\beta)^{1 / \beta-1} g(t) / c_{i}^{1 / \beta-1}(t) ;
$$

i.e., (33) holds.

Step 4. Now, let Assumption 5.1 hold and let both players operate on the market. Then,

$$
\pi\left(y \mid(t)=\left[g(t) /\left(y_{1}+y_{2}\right)\right]^{\beta},\right.
$$

and for the payoff to player $i$, we have

$$
p_{i}\left(y_{1}, y_{2} \mid t\right)=e^{-\lambda_{l}}\left[g(t)^{\beta} y_{i} /\left(y_{1}+y_{2}\right)^{\beta}-c_{i}(t) y_{i}\right] .
$$

Let us show that the instantaneous supply game has a unique Nash equilibrium under Assumption 5.1.

Since $p_{i}\left(y_{1}, y_{2} \mid t\right), i=1,2$, is strictly concave in $y_{i}$, a point $\left(y_{1}, y_{2}\right)$ is a Nash equilibrium if and only if

$$
\partial p_{i}\left(y_{1}, y_{2} \mid t\right) / \partial y_{i}=0
$$

or explicitly,

$$
g^{\beta}(t) / y^{\beta}-\beta g^{\beta}(t) y_{i} / y^{\beta+1}-c_{i}(t)=0 .
$$

Here, as above,

$$
y=y_{1}+y_{2} .
$$

For the sum of the left-hand sides for $i=1,2$, we have

$$
2 g^{\beta}(t) / y^{\beta}-\beta g^{\beta}(t) / y^{\beta}-\left[c_{1}(t)+c_{2}(t)\right]=0 .
$$

Hence,

$$
(2-\beta) g^{\beta}(t)=\left[c_{1}(t)+c_{2}(t)\right] y^{\beta}
$$

and

$$
y^{\beta}=(2-\beta) g^{\beta}(t) /\left[c_{1}(t)+c_{2}(t)\right] .
$$

Rewriting (56) as

$$
\beta g^{\beta}(t) y_{i}=g^{\beta}(t) y-c_{i}(t) y^{\beta+1}
$$


and using (57), we get

$$
\begin{aligned}
y_{i} & =\left[y / \beta g^{\beta}(t)\right]\left[g^{\beta}(t) \cdots c_{i}(t) y^{\beta}\right] \\
& =\left[y / \beta g^{\beta}(t)\right]\left(g^{\beta}(t) \cdots\left\{(2 \cdots \beta) c_{i}(t) /\left[c_{1}(t)+c_{2}(t)\right]\right\} g^{\beta}(t)\right) \\
& =\left\{(2-\beta) g^{\beta}(t) /\left[c_{1}(t)+c_{2}(t)\right]\right\}^{1 / \beta}(1 / \beta)\left\{1-(2-\beta) c_{i}(t) /\left[c_{1}(t)+c_{2}(t)\right]\right\} \\
& =\left[(2-\beta)^{1 / \beta} / \beta\right]\left\{1 \cdots(2-\beta) c_{i}(t) /\left[c_{1}(t)+c_{2}(t)\right]\right\} g(t) /\left[c_{1}(t)+c_{2}(t)\right]^{1 / \beta} .
\end{aligned}
$$

The latter is necessary for $\left(y_{1}, y_{2}\right)$ to be a Nash equilibrium in the supply game. Hence, if the Nash equilibrium exists, it is unique. Point $\left(y_{1}, y_{2}\right)$ given by (58) has positive components due to Assumption 5.1; See (28). Moreover, $\left(y_{1}, y_{2}\right)$ satisfies (56), where $y=y_{1}+y_{2}$, which is equivalent to (55). Hence, $\left(y_{1}, y_{2}\right)$ is the Nash equilibrium. We have stated that a unique Nash equilibrium exists. Denote it $\left(y_{1}(t), y_{2}(t)\right)$. By (58), we get

$$
y_{i}(t)=\left[(2-\beta)^{1 / \beta} / \beta\right]\left\{1-(2-\beta) c_{i}(t) /\left[c_{1}(t)+c_{2}(t)\right]\right\} g(t) /\left[c_{1}(t)+c_{2}(t)\right]^{1 / \beta} .
$$

By definition,

$$
b_{i 2}(t)=p_{i}\left(y_{1}(t), y_{2}(t) \mid t\right) .
$$

Substituting $y_{i}=y_{i}(t), i=1,2$, into (54) and noticing that

$$
y=y_{1}(t)+y_{2}(t)
$$

is given by (57), we get

$$
\begin{aligned}
b_{i 2}(t)= & e^{-\lambda t}\left[\frac{g^{\beta}(t)}{y^{\beta}} \cdots c_{i}(t)\right] y_{i}(t) \\
= & e^{-\lambda t}\left\{\left[c_{1}(t)+c_{2}(t)\right] /(2-\beta)-c_{i}(t)\right\} y_{i}(t) \\
= & e^{-\lambda t}\left\{\frac{\left[c_{1}(t)+c_{2}(t)\right]}{(2-\beta)}\right\}\left(1-\frac{(2-\beta) c_{i}(t)}{c_{1}(t)+c_{2}(t)}\right)\left[(2-\beta)^{1 / \beta} / \beta\right] \times \\
& \left\{1-(2-\beta) c_{i}(t) /\left[c_{1}(t)+c_{2}(t)\right]\right\} \frac{g(t)}{\left[c_{1}(t)+c_{2}(t)\right]^{1 / \beta}},
\end{aligned}
$$

and finally,

$b_{i 2}(t)=e^{-\lambda t}(2-\beta)^{1 / \beta-1}\left\{1-(2-\beta) c_{i}(t) /\left[c_{1}(t)+c_{2}(t)\right]\right\}^{2} g(t) /\left[c_{1}(t)+c_{2}(t)\right]^{1 / \beta-1}$.

Formula (34) is proved. 
Step 5. By definition, we have

$$
\begin{aligned}
b_{i 2}(t) & =p_{i}\left(y_{1}(t), y_{2}(t) \mid t\right) \\
& =e^{-\lambda i}\left[\pi\left(t, y_{1}(t)+y_{2}(t)\right)-c_{i}(t)\right] y_{i}(t) \\
& =\left\{g(t) /\left[y_{1}(t)+y_{2}(t)\right]\right\}^{\beta} y_{i}(t)-c_{i}(t) y_{i}(t) \\
& <\left[g(t) / y_{i}(t)\right]^{\beta} y_{i}(t)-c_{i}(t) y_{i}(t) \\
& \leq \sup _{y_{i}>0}\left\{\left[g(t) / y_{i}\right]^{\beta} y_{i}-c_{i}(t) y_{i}\right\} \\
& =b_{i 1}(t) .
\end{aligned}
$$

Inequality (35) is stated. Proposition 5.1 is proved.

Proof of Proposition 5.2. Let us check Assumption 2.1. The function $C_{i}\left(t_{i}\right)$ [see (29)] is continuously differentiable. The expression (52) for $C_{i}^{\prime}(t)$ shows that $C_{i}^{\prime}(t)<0$. Hence, $C_{i}(t)$ is monotonically decreasing. Consider the ratio in the right-hand side. The numerator $e^{-\lambda t_{i}} \bar{x}_{i}$ decreases in $t_{i}$ and the denominator $\left(1 \cdots e^{-\rho t_{i}}\right)^{\alpha}$ increases in $t_{i}$. Hence, the ratio decreases in $t_{i}$. Since the square bracket decreases in $t_{i}$, its product with the ratio decreases in $t_{i}$. As a result, we conclude that $C_{i}^{\prime}\left(t_{i}\right)$ increases in $t_{i}$. We have shown that Assumption 2.1 is satisfied.

Let us turn to Assumption 2.2. For the rate of cost reduction, we have the expression (31) whose denominator tends to 0 when $t$ approaches 0 . Hence, $a_{i}(t)$ tends to infinity as $t$ approaches 0 . Therefore, for all $t>0$ sufficiently small, we have

$$
a_{i}(t)>b_{i 1}(t)>b_{i 2}(t) \text {. }
$$

The expression for $a_{i}(t)$ and $b_{i 2}(t)$ [see (34)] show that

$$
b_{i 2}(t) / a_{i}(t)=h_{0}(t) h_{i 2}(t),
$$

where $h_{i 2}(t)$ is given in (36) and $h_{i}(t)$ is such that, for some $\tau>0$ and $\epsilon>0$, the lower bound inf $l_{l \geq \tau} h_{0}(t)>\epsilon$ holds. By Assumption 5.2, $h(t)$ tends to infinity as $t$ tends to infinity. Therefore, for all $t$ sufficiently large, we have

$$
b_{i 1}(t)>b_{i 2}(t)>a_{i}(t) \text {. }
$$

Since the functions $a_{i}(t), b_{i 1}(t), b_{i 2}(t)$ are continuous, there exist a $t_{i}^{-}>0$ that solves the equation

$$
a_{i}(t)=b_{i 1}(t)
$$

and $t_{i}^{+}>0$ that solves the equation

$$
a_{i}(t)=b_{i 2}(t) \text {. }
$$


In order to state that Assumption 2.2 holds, it is now sufficient to show that $t_{i}^{-}$and $t_{i}^{+}$are unique. We specify equation (59) by substituting the expressions for $a_{i}(t)$ and $b_{i 1}(t)$; see (31) and (33). We get

$$
\rho^{\alpha-1} \bar{x}_{i}^{\alpha} e^{-\lambda t}\left(\lambda+v \bar{x}_{i}^{\alpha} e^{-\rho t}\right) /\left(1-e^{-\rho t}\right)^{\alpha}=e^{-\lambda t}(1-\beta)^{1 / \beta-1} g(t) / c_{i}^{1 / \beta-1}(t) .
$$

Cancelling $e^{-\lambda t}$ and using the definition of $h_{i 1}(t)$ [see (36)], we arrive at equation (40). The right-hand side of (40) strictly increases in $t$ due to Assumption 5.2. Hence, equation (59) has the unique root $t_{i}$.

For equation (60), we argue similarly. Specify (60) by substituting the expression for $a_{i}(t)$ and $\mathrm{b}_{i 1}(t)$; see (31) and (34). We get

$$
\begin{aligned}
& \rho^{\alpha-1} \bar{x}_{i}^{\alpha} e^{-\cdots \hat{\lambda} t}\left(\lambda+v e^{-\rho t}\right) /\left(1-e^{-\rho t}\right)^{\alpha} \\
& =e^{-\lambda t}(2-\beta)^{1 / \beta-1}\left\{1-(2-\beta) c_{i}(t) /\left[c_{1}(t)+c_{2}(t)\right]\right\}^{2} g(t) /\left[c_{1}(t)+c_{2}(t)\right]^{1 / \beta-1} .
\end{aligned}
$$

Using the definition of $h_{i 2}(t)$ [see (36)], we arrive at equation (41). The righthand side of (41) strictly increases in $t$ due to Assumption 5.2. Hence, equation (60) has the unique root $t_{i}^{+}$.

Proposition 5.2 is proved.

Proof of Proposition 5.3. Let $i=1$ (for $i=2$, the argument is similar). Using formula (42) for $P_{1}\left(t_{1}, t_{2}\right)$ and taking into account that $\hat{t}_{2}$ lies between $t_{i}^{-}$and $t_{i}^{+}$(see Proposition 3.3), we specify the equality

$$
P_{1}\left(t_{1}^{-}, \hat{t}_{2}\right)=P_{1}\left(t_{1}^{+}, \hat{t}_{2}\right)
$$

into

$$
\begin{aligned}
& -\rho^{\alpha-1} e^{-\lambda t_{1}} \bar{x}_{1}^{\alpha} /\left(1-e^{-\rho t_{i}}\right)^{\alpha-1}+b_{11}^{0} e^{-\psi t t_{1}} / \psi+\left(b_{12}^{0}-b_{11}^{0}\right) e^{-\psi \hat{t}_{2}} / \psi \\
& =-\rho^{\alpha-1} e^{-\lambda t_{1}^{+}} \bar{x}_{1}^{\alpha} /\left(1 \cdots e^{-\rho t_{1}^{+}}\right)^{\alpha-1}+b_{12}^{0} e^{-\psi t_{1}^{+}} / \psi .
\end{aligned}
$$

Resolving with respect to $\hat{t}_{2}$, we get

$$
\begin{aligned}
\left(b_{12}^{0}-b_{11}^{0}\right) e^{-\psi \hat{i}_{2}} / \psi= & -\rho^{\alpha-1} e^{-\lambda r_{1}^{+}} \bar{x}_{1}^{\alpha} /\left(1-e^{-\alpha l_{1}^{+}}\right)^{\alpha-1}+b_{12}^{0} e^{-\psi t_{1}^{+}} / \psi \\
& +\rho^{\alpha-1} e^{-\lambda r_{1}} \bar{x}_{1}^{\alpha} /\left(1-e^{-\rho r_{1}}\right)^{\alpha-1}-b_{11}^{0} e^{-\psi r_{1}} / \psi,
\end{aligned}
$$

or

$$
\hat{t}_{2}=-(1 / \psi) \log \left[\psi G_{1} /\left(b_{12}^{0}-b_{11}^{0}\right)\right],
$$

where

$$
\begin{aligned}
G_{1}= & -\rho^{\alpha-1} e^{-\lambda t_{1}^{*}} \bar{x}_{1}^{\alpha} /\left(1-e^{-\rho t_{1}^{+}}\right)^{\alpha-1} \\
& +b_{12}^{0} e^{-\psi t_{1}^{+}} / \psi+\rho^{\alpha-1} e^{-\lambda r_{1}} \bar{x}_{1}^{\alpha} /\left(1-e^{-\rho t_{1}}\right)^{\alpha-1}-b_{11}^{0} e^{-\psi r_{1}^{+}} / \psi .
\end{aligned}
$$

The representation (44), (45) is stated. 


\section{Proof of Proposition 5.4.}

Step 1. Due to the form of $g(t)$ and $c_{i}(t)$ [see (37)], equation (40), which determines the fast choice $t_{i}^{-}$of player $i$, is specified as

$$
\rho^{\alpha-1} \bar{x}_{i}^{\alpha} /(1-\beta)^{1 / \beta-1}=\left[\left(1-e^{-\rho t}\right)^{\alpha} /\left(\lambda+v e^{-p t}\right)\right] g^{0} e^{k t} /\left(c_{i}^{0}\right)^{1 / \beta-1},
$$

or

$$
\rho^{\alpha-1} \bar{x}_{i}^{\alpha}\left(c_{i}^{0}\right)^{1 / \beta-1} /(1-\beta)^{1 / \beta-1} g^{0}=e^{\kappa t}\left(1 \cdots e^{-\rho t}\right)^{\alpha} /\left(\lambda+v e^{-\rho t}\right) .
$$

Using the notations (47) and (48), we arrive at equation (46).

Step 2. Due to (37), equation (41) determining $t_{i}^{+}$is specified as

$$
\begin{aligned}
& \rho^{\alpha-1} \bar{x}_{i}^{\alpha} /(1-\beta)^{1 / \beta-1} \\
& =\left[\left(1-e^{-\rho t}\right)^{\alpha} /\left(\lambda+v e^{-\rho t}\right)\right]\left[1-(2-\beta) c_{i}^{0} /\left(c_{1}^{0}+c_{2}^{0}\right)\right]^{2} g^{0} e^{\kappa t} /\left(c_{1}^{0}+c_{2}^{0}\right)^{1 / \beta-1} .
\end{aligned}
$$

Using the notations (47) and (50), we arrive at equation (49).

\section{Conclusions}

The paper is devoted to the analysis of a two-player game, in which the players strategies are the times of terminating the individual dynamical processes. The formal setting is related to the management of large-scale innovation projects, whose key feature is that the profits gained through the implementation of the projects are highly sensitive to the projects commercialization times. The basic reason for that is that the price formation mechanism changes rapidly the price as a new project is commercialized and the supply sharply increases. This situation is analyzed in the context of the competition of two projects on the construction of gas pipelines. In the game between the projects, the total profits gained during the pipelines life periods act as payoffs and the commercialization times as strategies. The reduction of project management to choices of the commercialization times is justified by the assumption that the individual regulation mechanisms, comprising investments into the construction of the gas pipelines and regulation of supply, work optimally provided the commercialization times are given. The analysis of the game leads to the restriction of the player rational choices to no more than two prescribed combinations of commercialization times, which constitute the Nash equilibria in the game. Typically, two Nash equilibria arise and the projects compete for a fast commercialization scenario; its complement, a slow commercialization scenario, is less profitable, representing the best response to the fast scenario of the competitor. A simple algorithm for finding the Nash equilibria is described. 


\section{References}

1. Klaassen, G., Roenrl, R. A., and Tarasyev, A. M., The Great Caspian Pipeline Game, Risk Management: Modeling and Computer Applications, Edited by V. Maksimov, Y. Ermoliev, and J. Linnerooth-Bayer, International Institute for Applied Systems Analysis, Laxenburg, Austria; IIASA Interim Report IR-01-066, pp. 107-131, 2001.

2. Arrow, K. J., and Kurz, M., Public Investment, the Rate of Return, and Optimal Fiscal Policy, Johns Hopkins University Press, Baltimore, Maryland, 1970.

3. Intriligator, M., Mathematical Optimization and Economic Theory, PrenticeHall, New York, NY, 1971.

4. Hofbaler, I., and Sigmund, K., The Theory of Evolution and Dynamic Systems, Cambridge University Press, Cambridge, England, 1988.

5. Friedman, D., Evolutionary Games in Economics, Econometrica, Vol. 59, pp. 637-666, 1991.

6. K.aniovski, Yu. M., Kryazhimski, A. V., and Young, H. P., Adaptive Dynamics in Games Played by Heterogeneous Populations, Games and Economic Behavior, Vol. 31, pp. 50-.96, 2000.

7. Kryazhimskir, A. V., and Osipov, Yu. S., On Evolutionary Differential Games, Proceedings of the Steklov Institute of Mathematics, Moscow, Russia, Vol. 211, pp. 257-287, 1995.

8. Kryazhimskil, A., Nentjes, A., Shibayev, S., and Tarasyev, A., Modeling Market Equilibrium for Transboundary Environmental Problems, Nonlinear Analysis: Theory, Methods, and Applications, Vol. 42, pp. 991-1002, 2001.

9. Tarasyev, A. M., Control Synthesis in Grid Schernes for Hanilton-Jacobi Equations, Annals of Operations Research, Vol. 88, pp. 337-359, 1999.

10. Basar, T., and Olsder, G. J., Dynamic Noncooperative Game Theory, Academic Press, London, England, 1982.

11. Vorob'Ey, N. N., Game Theory Lectures for Economists and Systems Scientists, Springer, New York, NY, 1977.

12. Klaassen, G., MCDonald, A., and Zhao, J., The Future of Gas Infrastructures in Eurasia, Energy Policy, Vol. 29, pp. 399-413, 2001.

13. Barzel, Y., Optimal Timing of Innovations, Review of Economics and Statistics, Vol. 50, pp. 348-355, 1968.

14. Tarasyev, A. M., and Watanabe, C., Dynamic Optimality Principles and Sensitivity Analysis in Models of Economic Growth, Nonlinear Analysis: Theory, Methods, and Applications, Vol. 47, pp. 2309-2320, 2001.

15. Pontryagin, L. S., Boltyanskil, V. G., Gamkrelidze, R. V., and Mishchenko, E. F., The Mathematical Theory of Optimal Processes, Interscience (Wiley), New York, NY, 1962.

16. Krasovskit, N. N., and Subbotin, A. I., Game-Theoretical Control Problems, Springer, New York, NY, 1988.

17. Chernousko, F. L., State Estimation for Dynamic Systemss, CRC Press, Boca Raton, Florida, 1994. 
